\title{
Highly Enantioselective Amination of $\alpha$-Substituted $\alpha$-Cyanoacetates with Chiral Catalysts Accessible from Both Quinine and Quinidine
}

\author{
Xiaofeng Liu, Hongming Li and Li Deng* \\ Department of Chemistry, Brandeis University, Waltham, Massachusetts 02454-9110 \\ *To whom correspondence should be addressed
}

Supporting Information

General Information. ${ }^{1} \mathrm{H}$ and ${ }^{13} \mathrm{C}$ NMR spectra were recorded on a Varian instrument (400 $\mathrm{MHz}$ and $100 \mathrm{MHz}$, respectively) and internally referenced to tetramethylsilane signal or the residual proton solvent signals. Infrared spectra were recorded on a Perkin Elmer FT-IR Spectrometer and are reported in frequency of absorption. High resolution mass spectra for all the new compounds were done by either a Micromass 70-VSE instrument $(\mathrm{CI})$ or a Micromass Q-Tof instrument (ESI). Specific rotations were measured on a Jasco Digital Polarimeter.

High performance liquid chromatography (HPLC) analysis was performed on a Hewlett-Packard 1100 Series instrument equipped with a quaternary pump, using a Daicel Chiralcel OD Column (250 x $4.6 \mathrm{~mm})$, Chiralpak AS Column $(250 \times 4.6 \mathrm{~mm})$ or Chiralpak AD Column ( $250 \times 4.6 \mathrm{~mm})$. UV detection was monitored at $220 \mathrm{~nm}$.

Ethyl $\alpha$-methyl- $\alpha$-cyanoacetate was purchased from TCI and was used as received. Ethyl $\alpha$-phenyl- $\alpha$-cyanoacetate and all dialkyl azodicarboxylate were purchased from Lancaster and were used as received. Other ethyl $\alpha$-aryl- $\alpha$-cyanoacetates were prepared according to literature procedures. ${ }^{1,2}$ All solvents were freshly distilled from sodium or $\mathrm{CaH}_{2}$ before use.

\section{Preparation of Q-Bn:}

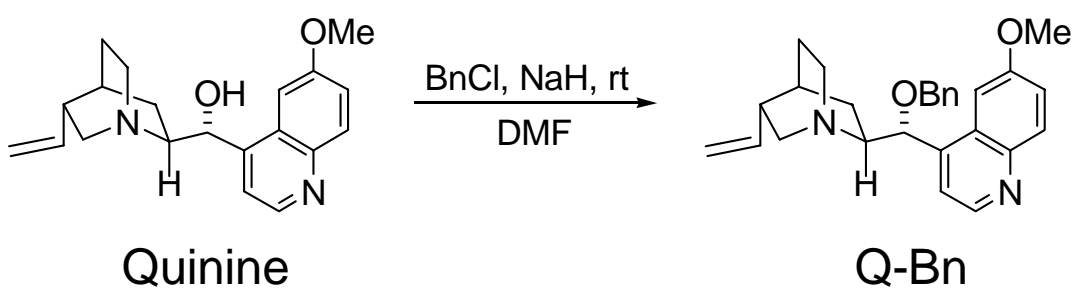

To a solution of quinine $(4.0 \mathrm{~g}, 12.4 \mathrm{mmol})$ in DMF $(40 \mathrm{~mL})$ under nitrogen atmosphere, $\mathrm{NaH}$ (1.36 g, 57\% suspension in mineral oil, $32.3 \mathrm{mmol}$ ) was added in small portions and the resulting mixture was stirred at room temperature for $2 \mathrm{~h}$. $\mathrm{BnCl}(1.56 \mathrm{~mL}, 13.6 \mathrm{mmol})$ was added dropwise via a syringe in $10 \mathrm{~min}$. The mixture was stirred at room temperature overnight. After the starting material was completely consumed, brine $(40 \mathrm{~mL})$ was added carefully and the resulting mixture was extracted with ethyl acetate $(200 \mathrm{~mL})$. The organic phase was washed with $\mathrm{H}_{2} \mathrm{O}(5 \times 100 \mathrm{~mL})$, brine $(100 \mathrm{~mL})$ and dried over $\mathrm{Na}_{2} \mathrm{SO}_{4}$. The solvent was removed in vacuo to afford a light yellow oil $(5.1 \mathrm{~g}, 99 \%)$. This crude product (Q-Bn) was used without further purification. 


\section{Preparation of Q-2c:}
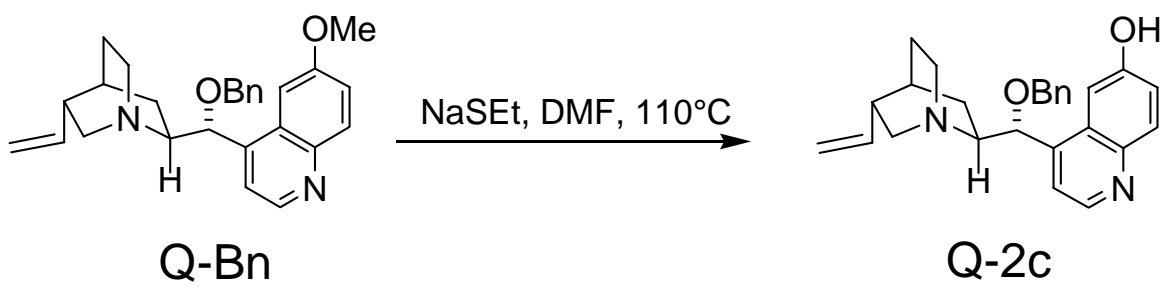

Under $\mathrm{N}_{2}$ atmosphere, a suspension of Q-Bn (5.1 g, $\left.12.3 \mathrm{mmol}\right)$ and NaSEt (4.2 g, 50.0 mmol) in dry DMF $(75 \mathrm{~mL})$ was stirred at $110{ }^{\circ} \mathrm{C}$ for 9 hours. The reaction mixture was cooled to room temperature, and the reaction was quenched with sat. $\mathrm{NH}_{4} \mathrm{Cl}(80 \mathrm{ml})$ and $\mathrm{H}_{2} \mathrm{O}(60 \mathrm{~mL})$. The solution was acidified to $\mathrm{pH}=2$ by conc. $\mathrm{HCl}$. This aqueous solution was washed by ethyl acetate $(2 \times 100 \mathrm{~mL})$ and its $\mathrm{pH}$ value was adjusted to 8 by conc. ammonium hydroxide. The resulting mixture was extracted with ethyl acetate $(2 \times 150$ $\mathrm{mL}$ ). The combined organic phase was dried over $\mathrm{Na}_{2} \mathrm{SO}_{4}$, and concentrated in vacuo to afford a yellowish solid. This solid was washed by $\mathrm{CH}_{2} \mathrm{Cl}_{2}(2 \times 30 \mathrm{~mL})$ and then dissolved in aqueous $\mathrm{HCl}(2 \mathrm{~N}, 150 \mathrm{~mL})$. The resulting solution was washed by ethyl acetate $(50$ $\mathrm{mL}$ ) and adjusted to $\mathrm{pH}=7$ by conc. ammonium hydroxide. The aqueous phase was extracted by ethyl acetate $(300 \mathrm{~mL})$. The organic phase was dried quickly with $\mathrm{Na}_{2} \mathrm{SO}_{4}$. After concentration, Q-2c was obtained as a white powder $(3.764 \mathrm{~g}, 77 \%)$. $[\alpha]_{\mathrm{D}}{ }^{23}=-78.9$ (c 0.98, EtOH); ${ }^{1} \mathrm{H}$ NMR (400 MHz, DMSO-d 6 ) $\delta(\mathrm{ppm}) 10.03(\mathrm{br}, 1 \mathrm{H}), 8.63(\mathrm{~d}, \mathrm{~J}=4.4$ $\mathrm{Hz}, 1 \mathrm{H}), 7.89(\mathrm{~d}, \mathrm{~J}=9.2 \mathrm{~Hz}, 1 \mathrm{H}), 7.70-7.15(\mathrm{~m}, 8 \mathrm{H}), 5.92-5.77(\mathrm{~m}, 1 \mathrm{H}), 5.14-4.82(\mathrm{~m}$, $3 \mathrm{H}), 4.34(\mathrm{~d}, \mathrm{~J}=11.6 \mathrm{~Hz}, 1 \mathrm{H}), 4.27$ (d, J = 11.6 Hz, 1H), 3.30-2.94 (m , 2H), 2.88-2.70 $(\mathrm{m}, 1 \mathrm{H}), 2.50-2.10(\mathrm{~m}, 3 \mathrm{H}), 1.94-1.32(\mathrm{~m}, 5 \mathrm{H}) ;{ }^{13} \mathrm{C}$ NMR $\left(100 \mathrm{MHz}, \mathrm{DMSO}-\mathrm{d}_{6}\right) \delta(\mathrm{ppm})$ $155.4,146.6,144.3,143.4,142.3,138.1,131.3,128.2,127.7,127.6,127.5,121.4,114.2$, $104.7,70.5,59.9,56.0,54.9,41.7,39.4,27.4,27.3,24.6$; IR (KBr) v (cm $\left.{ }^{-1}\right) 3395,3063$, 3033, 2953, 2908, 2863, 2753, 2686, 2613, 2539, 1906, 1638, 1613, 1591, 1509, 1499, $1463,1401,1355,1331,1280,1241,1215,1132,1100,1030,1014,921,853,814,751$, 702; HRMS (ESI) m/z calcd for $\left(\mathrm{C}_{26} \mathrm{H}_{29} \mathrm{~N}_{2} \mathrm{O}_{2}+\mathrm{H}^{+}\right)$: 401.2229, found: 401.2228 .

\section{General Procedure for Enantioselective Amination of Ethyl a-Substituted a- Cyanoacetate with Dialkyl Azodicarboxylate}

\section{Reaction Catalyzed by QD-2c:}

To a solution of ethyl $\alpha$-substituted $\alpha$-cyanoacetate $(0.22 \mathrm{mmol}, 1.1 \mathrm{eq})$ and QD-2c (4 mg or $8 \mathrm{mg}, 5 \mathrm{~mol} \%$ or $10 \mathrm{~mol} \%$ ) in toluene $\left(2 \mathrm{~mL}\right.$ ) at $-78{ }^{\circ} \mathrm{C}$ (or at RT) under stirring, a solution of di-tert-butyl azodicarboxylate or dibenzyl azodicarboxylate in toluene $(0.4$ $\mathrm{mL}, 0.5 \mathrm{M}, 0.2 \mathrm{mmol}, 1.0 \mathrm{eq})$ was added dropwise within 5-10 min. The reaction mixture was stirred until the color of the solution turned from yellow to colorless ( $1 \mathrm{~min}-8 \mathrm{~h})$, after which it was allowed to warm to room temperature and the pure product was isolated by flash chromatography. 


\section{Reaction Catalyzed by Q-2c:}

A suspension of Q-2c (4 mg or $8 \mathrm{mg}, 5 \mathrm{~mol} \%$ or $10 \mathrm{~mol} \%)$ in toluene $(2 \mathrm{~mL})$ was subjected to ultrasound until no chunky solid was visible (c.a. $15 \mathrm{~min}$ ). The resulting mixture was heated to $110^{\circ} \mathrm{C}$ until a clear solution was formed (10-15 min). While it was still hot, the solution was allowed to pass a cotton plug to remove any trace amount of insoluble residue and then cooled to room temperature. Then ethyl $\alpha$-substituted $\alpha$ cyanoacetate $(0.22 \mathrm{mmol}, 1.1 \mathrm{eq})$ was added. This mixture was stirred and cooled to -78 ${ }^{\circ} \mathrm{C}$ (or at RT). A solution of di-tert-butyl azodicarboxylate or dibenzyl azodicarboxylate in toluene $(0.4 \mathrm{~mL}, 0.5 \mathrm{M}, 0.2 \mathrm{mmol}, 1.0 \mathrm{eq})$ was added dropwise within 5-10 min and stirring was continued until the color of the solution turned from yellow to colorless ( 1 min-12 h). The reaction mixture was allowed to warm to room temperature and the pure product was isolated by flash chromatography.

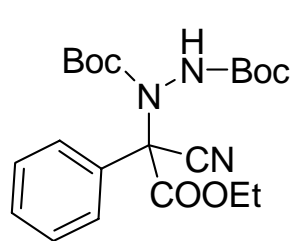

$(+)-5 a$. This reaction was catalyzed by Q-2c and the product was obtained as a white foam in $92 \%$ yield after purification by flash chromatography (hexanes/ether $=3 / 1$ ) and $97 \%$ ee as determined by HPLC analysis [Chiralpak AD, Hexanes:IPA, $85: 15,1.0 \mathrm{~mL} / \mathrm{min}, \lambda$ $220 \mathrm{~nm}, \mathrm{t}($ minor $)=10.7 \mathrm{~min}, \mathrm{t}($ major $)=32.3 \mathrm{~min}] .[\alpha]_{\mathrm{D}}^{23}=+64.0$ (c $\left.0.175, \mathrm{CHCl}_{3}\right)$; ${ }^{1} \mathrm{H}$ NMR $\left(400 \mathrm{MHz}, \mathrm{CD}_{3} \mathrm{CN}\right)$ see the attached spectrum; ${ }^{13} \mathrm{C} \mathrm{NMR}$ $\left(100 \mathrm{MHz}, \mathrm{CD}_{3} \mathrm{CN}\right)$ see the attached spectrum; IR $\left(\mathrm{CHCl}_{3}\right) \vee\left(\mathrm{cm}^{-1}\right) 3347,2980,1744$, $1718,1453,1368,1242,1154,1052$; HRMS (CI) m/z calcd. for $(\mathrm{M}+\mathrm{H})^{+}: 420.2135$, found 420.2132 .

(-)-5a. This reaction was catalyzed by QD-2c and the product was obtained as a white foam in $92 \%$ yield and $95 \%$ ee.

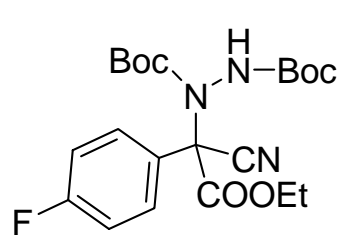

$(+)-5 b$. This reaction was catalyzed by Q-2c and the product was obtained as a white foam in $95 \%$ yield after purification by flash chromatography (hexanes/ether $=3 / 1$ ) and $96 \%$ ee as determined by HPLC analysis [Chiralpak AD, Hexanes:IPA, 85:15, $1.0 \mathrm{~mL} / \mathrm{min}, \lambda$ $220 \mathrm{~nm}, \mathrm{t}$ (minor) $=10.9 \mathrm{~min}, \mathrm{t}($ major $)=34.4 \mathrm{~min}] . \quad[\alpha]_{\mathrm{D}}^{23}=+$ 59.7 (c $\left.1.14, \mathrm{CHCl}_{3}\right) ;{ }^{1} \mathrm{H}$ NMR $\left(400 \mathrm{MHz}, \mathrm{CD}_{3} \mathrm{CN}\right)$ see the attached spectrum; ${ }^{13} \mathrm{C} \mathrm{NMR}$ $\left(100 \mathrm{MHz}, \mathrm{CD}_{3} \mathrm{CN}\right)$ see the attached spectrum; IR $\left(\mathrm{CHCl}_{3}\right) \vee\left(\mathrm{cm}^{-1}\right) 3348,2980,2929$, 1745, 1719, 1607, 1510, 1476, 1369, 1241, 1157, 1054, 1016; HRMS (ESI) m/z calcd. for $(\mathrm{M}+\mathrm{H})^{+}$: 438.2029, found 438.2024.

(-)-5b. This reaction was catalyzed by QD-2c and the product was obtained as a white foam in $97 \%$ yield and $94 \%$ ee.

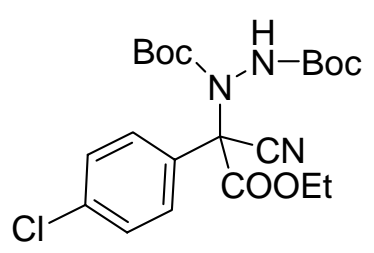

$(+)-5 c$. This reaction was catalyzed by Q-2c and the product was obtained as a white foam in $94 \%$ yield after purification by flash chromatography (hexanes/ether $=4 / 1$ ) and $97 \%$ ee as determined by HPLC analysis [Chiralpak AD, Hexanes:IPA, 85:15, 1.0 $\mathrm{mL} / \mathrm{min}, \lambda 220 \mathrm{~nm}, \mathrm{t}$ (minor) $=11.6 \mathrm{~min}, \mathrm{t}($ major $)=36.6 \mathrm{~min}]$. 
$[\alpha]_{\mathrm{D}}{ }^{23}=+61.6\left(\mathrm{c} 1.635, \mathrm{CHCl}_{3}\right) ;{ }^{1} \mathrm{H}$ NMR $\left(400 \mathrm{MHz}, \mathrm{CDCl}_{3}\right)$ see the attached spectrum; ${ }^{13} \mathrm{C}$ NMR $\left(100 \mathrm{MHz}, \mathrm{CDCl}_{3}\right)$ see the attached spectrum; IR $\left(\mathrm{CHCl}_{3}\right) \vee\left(\mathrm{cm}^{-1}\right)$ 3335, 2982, 2935, 1747, 1721, 1494, 1478, 1370, 1244, 1154, 1097, 1016.

(-)-5c. This reaction was catalyzed by QD-2c and the product was obtained as a white foam in $96 \%$ yield and $93 \%$ ee.

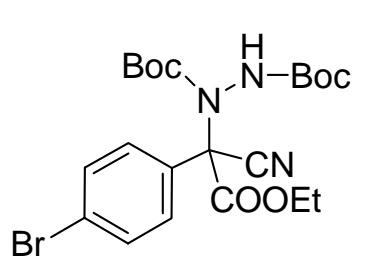

$(+)-5 d$. This reaction was catalyzed by Q-2c and the product was obtained as a white foam in $97 \%$ yield after purification by flash chromatography (hexanes/ether $=4 / 1$ ) and $96 \%$ ee as determined by HPLC analysis [Chiralpak AD, Hexanes:IPA, $85: 15,1.0 \mathrm{~mL} / \mathrm{min}$, $\lambda 220 \mathrm{~nm}, \mathrm{t}$ (minor) $=12.4 \mathrm{~min}, \mathrm{t}($ major $)=35.3 \mathrm{~min}] .[\alpha]_{\mathrm{D}}^{23}=+$ 54.4 (c $\left.0.54, \mathrm{CHCl}_{3}\right) ;{ }^{1} \mathrm{H}$ NMR $\left(400 \mathrm{MHz}, \mathrm{CD}_{3} \mathrm{CN}\right)$ see the attached spectrum; ${ }^{13} \mathrm{C}$ NMR $\left(100 \mathrm{MHz}, \mathrm{CD}_{3} \mathrm{CN}\right)$ see the attached spectrum; IR $\left(\mathrm{CHCl}_{3}\right)$ $v\left(\mathrm{~cm}^{-1}\right)$ 3422, 2980, 2932, 1745, 1718, 1644, 1488, 1369, 1243, 1154, 1012; HRMS (ESI) $\mathrm{m} / \mathrm{z}$ calcd. for $(\mathrm{M}+\mathrm{H})^{+}: 498.1240$, found 498.1253 .

(-)-5d. This reaction was catalyzed by QD-2c and the product was obtained as a white foam in $99 \%$ yield and $93 \%$ ee.

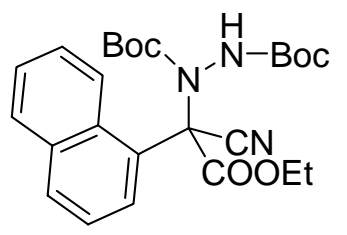

$(+)-5 e$. This reaction was catalyzed by Q-2c and the product was obtained as a white foam in $98 \%$ yield after purification by flash chromatography (hexanes/ether $=3 / 1$ ) and $99 \%$ ee as determined by HPLC analysis [Chiralpak AD, Hexanes:IPA, 60:40, $1.0 \mathrm{~mL} / \mathrm{min}, \lambda$ $220 \mathrm{~nm}, \mathrm{t}$ (minor $)=9.6 \mathrm{~min}, \mathrm{t}($ major $)=39.6 \mathrm{~min}] .[\alpha]_{\mathrm{D}}{ }^{23}=+63.9$ (c $\left.0.49, \mathrm{CHCl}_{3}\right) ;{ }^{1} \mathrm{H}$ NMR $\left(400 \mathrm{MHz}, \mathrm{CD}_{3} \mathrm{CN}\right)$ see the attached spectrum; ${ }^{13} \mathrm{C}$ NMR $(100$ $\left.\mathrm{MHz}, \mathrm{CD}_{3} \mathrm{CN}\right)$ see the attached spectrum; IR $\left(\mathrm{CHCl}_{3}\right) \vee\left(\mathrm{cm}^{-1}\right) 3440,2981,1721,1645$, 1476, 1369, 1244, 1152, 1015; HRMS (CI) m/z calcd. for $\left(\mathrm{M}+\mathrm{H}^{+}\right)$: 470.2291, found 470.2295 .

(-)-5e. This reaction was catalyzed by QD-2c and the product was obtained as a white foam in $99 \%$ yield and $96 \%$ ee.<smiles>CCOC(=O)C(C)(C)C(NN(C(=O)OCc1ccccc1)C(=O)OC(C)(C)C)(c1ccc(C)cc1)C(C)(C)C</smiles>

$(+)-5 f$. This reaction was catalyzed by Q-2c and the product was obtained as a white foam in $96 \%$ yield after purification by flash chromatography (hexanes/ether $=3 / 1$ ) and $96 \%$ ee as determined by HPLC analysis [Chiralpak AD, Hexanes:IPA, 85:15, 1.0 $\mathrm{mL} / \mathrm{min}, \lambda 220 \mathrm{~nm}, \mathrm{t}$ (minor) $=11.5 \mathrm{~min}, \mathrm{t}$ (major) $=31.6 \mathrm{~min}]$. $[\alpha]_{\mathrm{D}}^{23}=+70.8\left(\mathrm{c} 0.655, \mathrm{CHCl}_{3}\right) ;{ }^{1} \mathrm{H}$ NMR $\left(400 \mathrm{MHz}, \mathrm{CD}_{3} \mathrm{CN}\right)$ see the attached spectrum; ${ }^{13} \mathrm{C}$ NMR $\left(100 \mathrm{MHz}, \mathrm{CD}_{3} \mathrm{CN}\right)$ see the attached spectrum; IR $\left(\mathrm{CHCl}_{3}\right) \vee\left(\mathrm{cm}^{-1}\right) 3428,2980,2933,1745,1719,1649,1511,1476,1458,1369,1242$, $1155,1055,1018$; HRMS (CI) $\mathrm{m} / \mathrm{z}$ calcd. for $\left(\mathrm{M}+\mathrm{H}^{+}\right)$: 434.2291, found 434.2271.

(-)-5f. This reaction was catalyzed by QD-2c and the product was obtained as a white foam in $96 \%$ yield and $94 \%$ ee. 


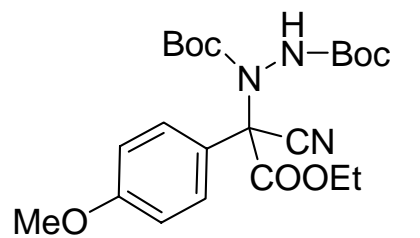
$[\alpha]_{\mathrm{D}}{ }^{23}=+70.0\left(\mathrm{c} 0.67, \mathrm{CHCl}_{3}\right) ;{ }^{1} \mathrm{H} \mathrm{NMR}\left(400 \mathrm{MHz}, \mathrm{CD}_{3} \mathrm{CN}\right)$ see the attached spectrum; ${ }^{13} \mathrm{C}$ NMR $\left(100 \mathrm{MHz}, \mathrm{CD}_{3} \mathrm{CN}\right)$ see the attached spectrum; IR $\left(\mathrm{CHCl}_{3}\right) \vee\left(\mathrm{cm}^{-1}\right) 3355$, 2980, 2935, 1744, 1718, 1610, 1512, 1460, 1369, 1245, 1155, 1055, 1031; HRMS (ESI) $\mathrm{m} / \mathrm{z}$ calcd. for $(\mathrm{M}+\mathrm{H})^{+}: 450.2240$, found 450.2250 .

(-)-5g. This reaction was catalyzed by QD-2c and the product was obtained as a white foam in $96 \%$ yield and $94 \%$ ee.

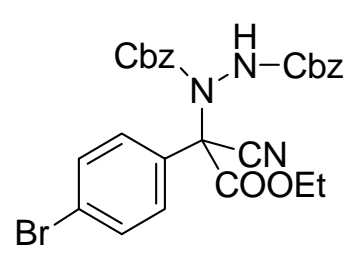

(+)-5h. This reaction was catalyzed by Q-2c and the product was obtained as a white foam in $83 \%$ yield after purification by flash chromatography (hexanes/ether $=3 / 1$ ) and $92 \%$ ee as determined by HPLC analysis [Chiralpak AS, Hexanes:IPA, 75:25, $1.0 \mathrm{~mL} / \mathrm{min}, \lambda$ $220 \mathrm{~nm}, \mathrm{t}$ (minor) $=10.6 \mathrm{~min}, \mathrm{t}$ (major) $=18.9 \mathrm{~min}] .[\alpha]_{\mathrm{D}}^{23}=+$ 45.2 (c $0.405, \mathrm{CHCl}_{3}$ ); ${ }^{1} \mathrm{H} \mathrm{NMR}\left(400 \mathrm{MHz}, \mathrm{CDCl}_{3}\right)$ see the attached spectrum; ${ }^{13} \mathrm{C} \mathrm{NMR}\left(100 \mathrm{MHz}, \mathrm{CDCl}_{3}\right)$ see the attached spectrum; IR $\left(\mathrm{CHCl}_{3}\right) \mathrm{v}$ $\left(\mathrm{cm}^{-1}\right) 3454,3033,2089,1749,1727,1650,1635,1395,1340,1229,1012$; HRMS (ESI) $\mathrm{m} / \mathrm{z}$ calcd. for $(\mathrm{M}+\mathrm{H})^{+}: 566.0927$, found 566.0916 .

(-)-5h. This reaction was catalyzed by QD-2c and the product was obtained as a white foam in $86 \%$ yield and $91 \%$ ee.

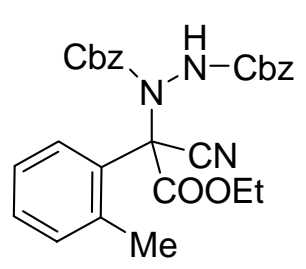

(+)-5i. This reaction was catalyzed by Q-2c and the product was obtained as a white foam in $72 \%$ yield after purification by flash chromatography (hexanes/ether $=2 / 1$ ) and $87 \%$ ee as determined by HPLC analysis [Chiralpak OD, Hexanes:IPA, 90:10, $1.0 \mathrm{~mL} / \mathrm{min}, \lambda$ $220 \mathrm{~nm}, \mathrm{t}$ (minor) $=42.7 \mathrm{~min}, \mathrm{t}$ (major) $=34.8 \mathrm{~min}] .[\alpha]_{\mathrm{D}}{ }^{23}=+18.9$ (c 2.73, $\left.\mathrm{CHCl}_{3}\right) ;{ }^{1} \mathrm{H}$ NMR $\left(400 \mathrm{MHz}, \mathrm{CD}_{3} \mathrm{CN}\right)$ see the attached spectrum; ${ }^{13} \mathrm{C}$ NMR $\left(100 \mathrm{MHz}, \mathrm{CD}_{3} \mathrm{CN}\right)$ see the attached spectrum; IR $\left(\mathrm{CHCl}_{3}\right) \vee\left(\mathrm{cm}^{-1}\right)$ 3318, 2924, 1732, 1495, 1455, 1391, 1333, 1227, 1092, 1023; HRMS (ESI) m/z calcd. for $(\mathrm{M}+\mathrm{H})^{+}:$:502.1978, found 502.1983.

(-)-5i. This reaction was catalyzed by QD-2c and the product was obtained as a white foam in $71 \%$ yield and $82 \%$ ee.

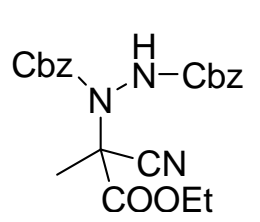

(-)-5j. This reaction was catalyzed by Q-2c and the product was obtained as a white foam in $74 \%$ yield after purification by flash chromatography (hexanes/ether $=2 / 1$ ) and $23 \%$ ee as determined by HPLC analysis [Chiralpak OD, Hexanes:IPA, 93:7, $1.0 \mathrm{~mL} / \mathrm{min}, \lambda 220 \mathrm{~nm}, \mathrm{t}$ (major) = $31.2 \mathrm{~min}, \mathrm{t}$ (minor) $=36.9 \mathrm{~min}] .[\alpha]_{\mathrm{D}}{ }^{23}=-0.9\left(\mathrm{c} 3.045, \mathrm{CHCl}_{3}\right) ;{ }^{1} \mathrm{H}$ NMR (400 MHz, $\left.\mathrm{CDCl}_{3}\right)$ see the attached spectrum; ${ }^{13} \mathrm{C} \mathrm{NMR}\left(100 \mathrm{MHz}, \mathrm{CDCl}_{3}\right)$ see the attached spectrum; IR $\left(\mathrm{CHCl}_{3}\right) \vee\left(\mathrm{cm}^{-1}\right) 3439,3033,2354,2066,1746,1720,1635,1499$, 
1454, 1395, 1347, 1288, 1233, 1174, 1123, 1049; HRMS (ESI) m/z calcd. for $(\mathrm{M}+\mathrm{H})^{+}$: 426.1665, found 426.1653 .

$(+)-5 j$. This reaction was catalyzed by QD-2c and the product was obtained as a white foam in $75 \%$ yield and $35 \%$ ee. 
HPLC Conditions: Daicel Chirapak AD, hexanes:IPA 85:15, $1.0 \mathrm{ml} / \mathrm{min}$.<smiles>CCOC(=O)C(C)(C)N(NC(=O)OC(C)(C)C)C(C)(C)C(=O)OC(C)(C)C</smiles>

Racemic 5a

(+)-5a

Product of Q-2c catalyzed reaction

$(-)-5 a$

Product of QD-2c catalyzed reaction
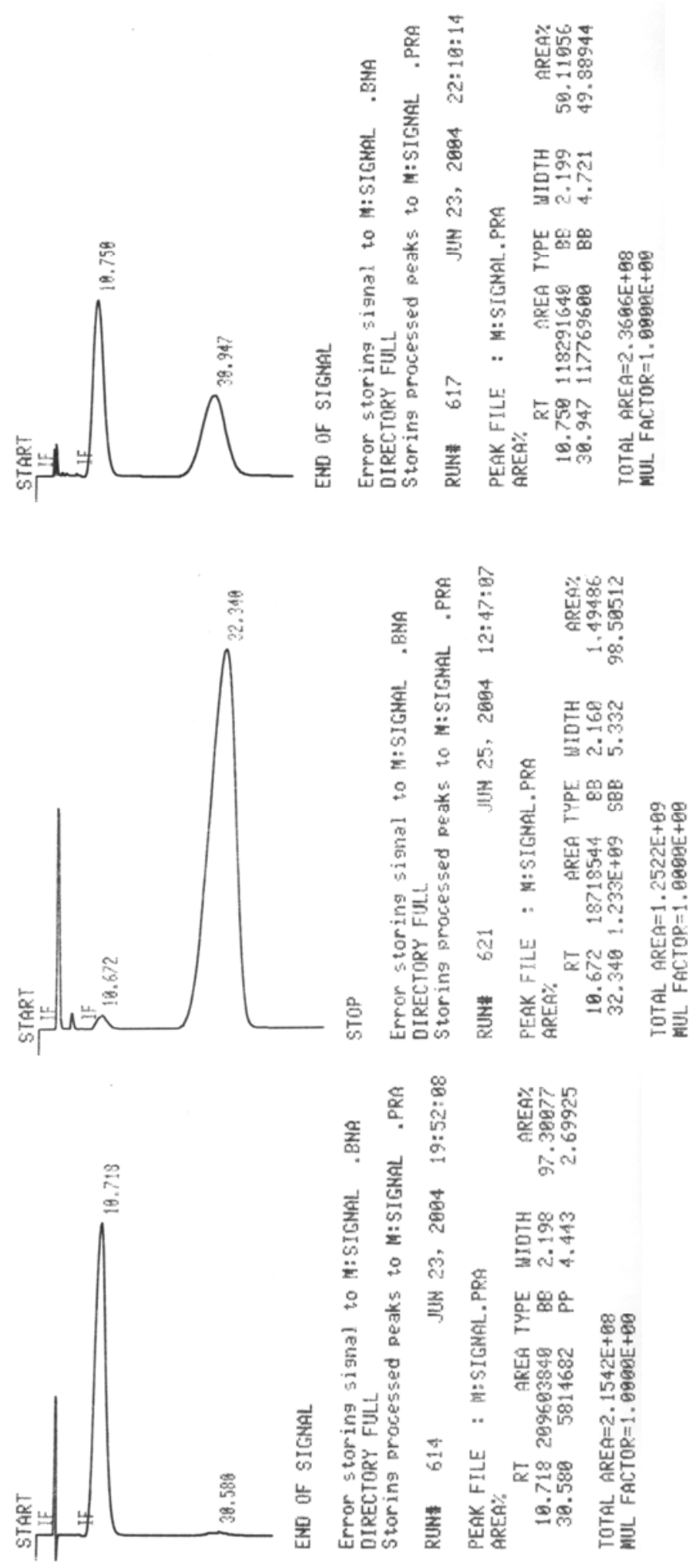


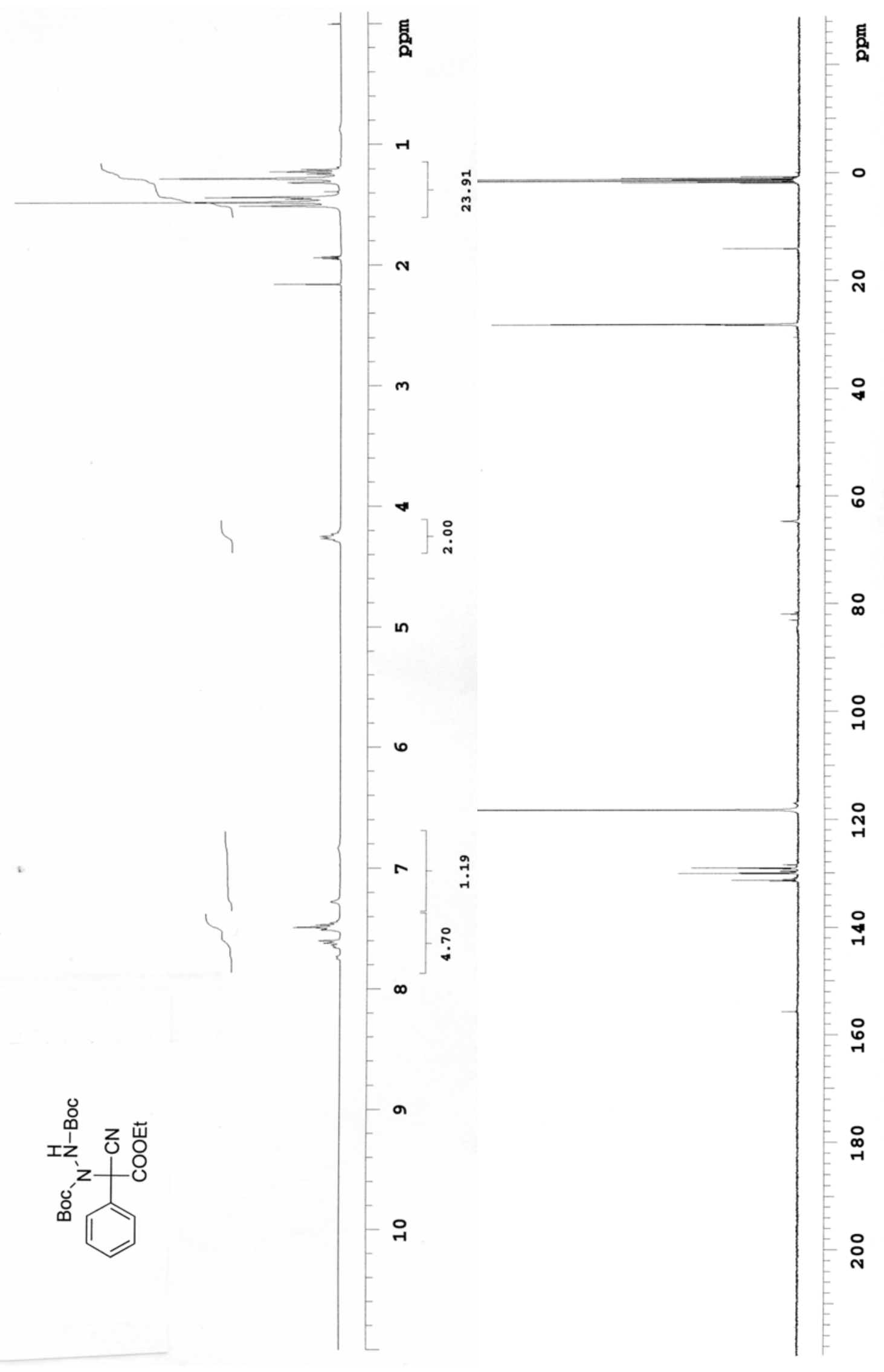


HPLC Conditions: Daicel Chirapak AD, hexanes:IPA 85:15, $1.0 \mathrm{ml} / \mathrm{min}$.
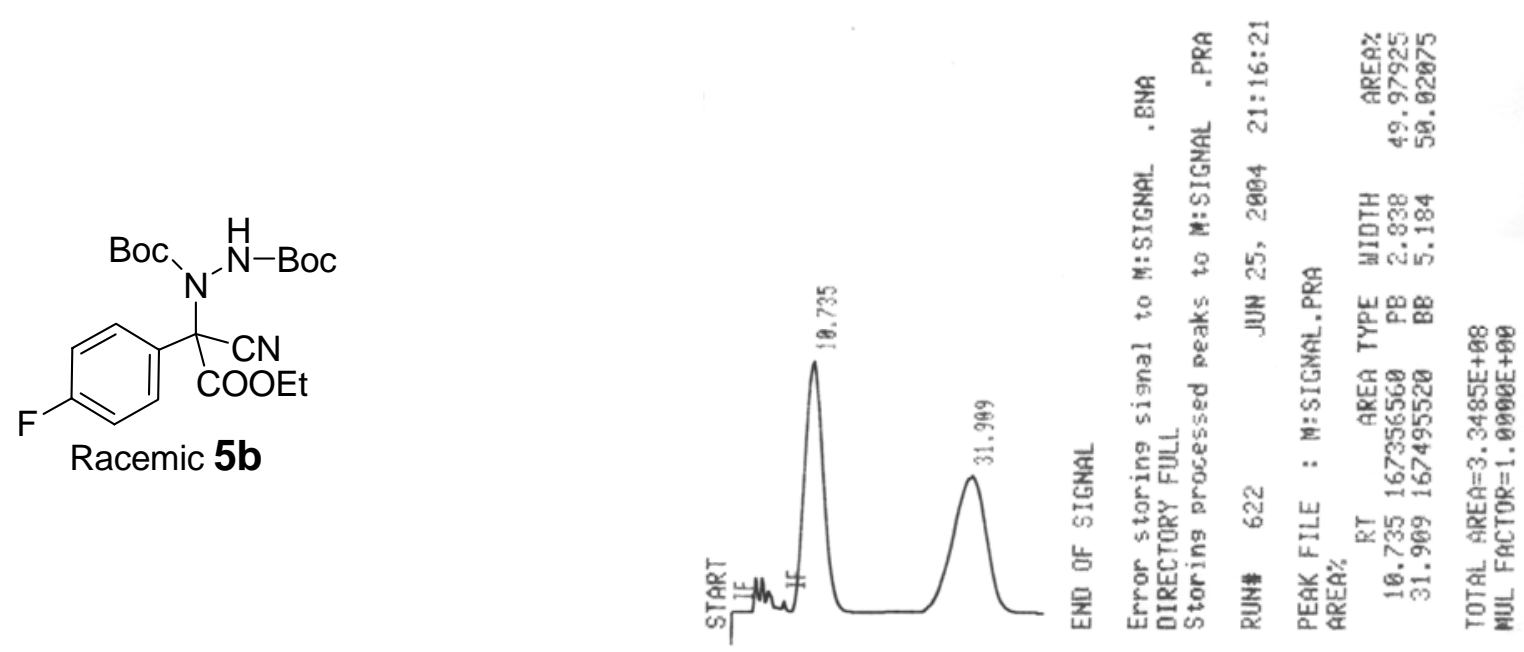

$(+)-5 b$

Product of Q-2c catalyzed reaction

$(-)-5 b$

Product of QD-2c

catalyzed reaction
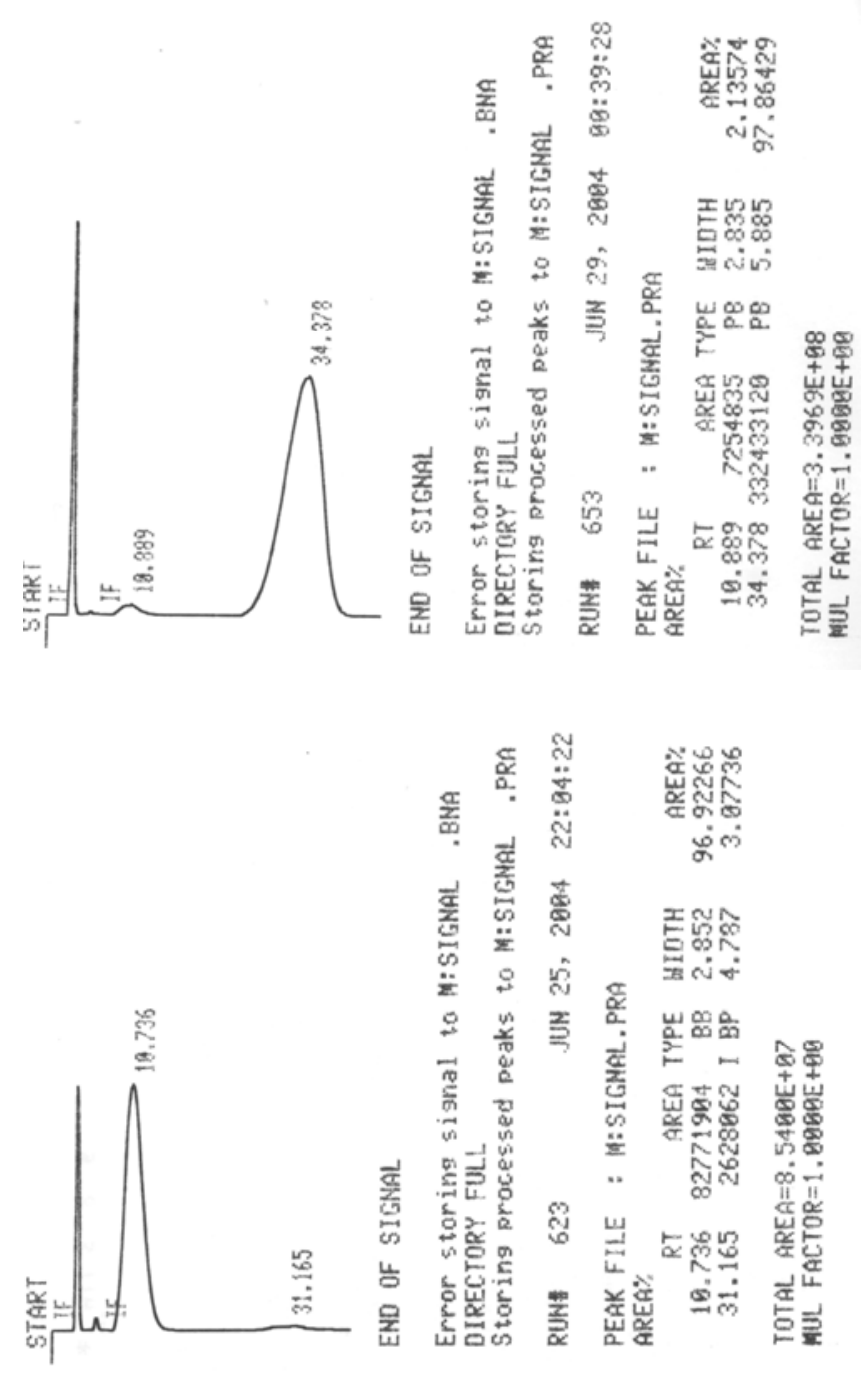


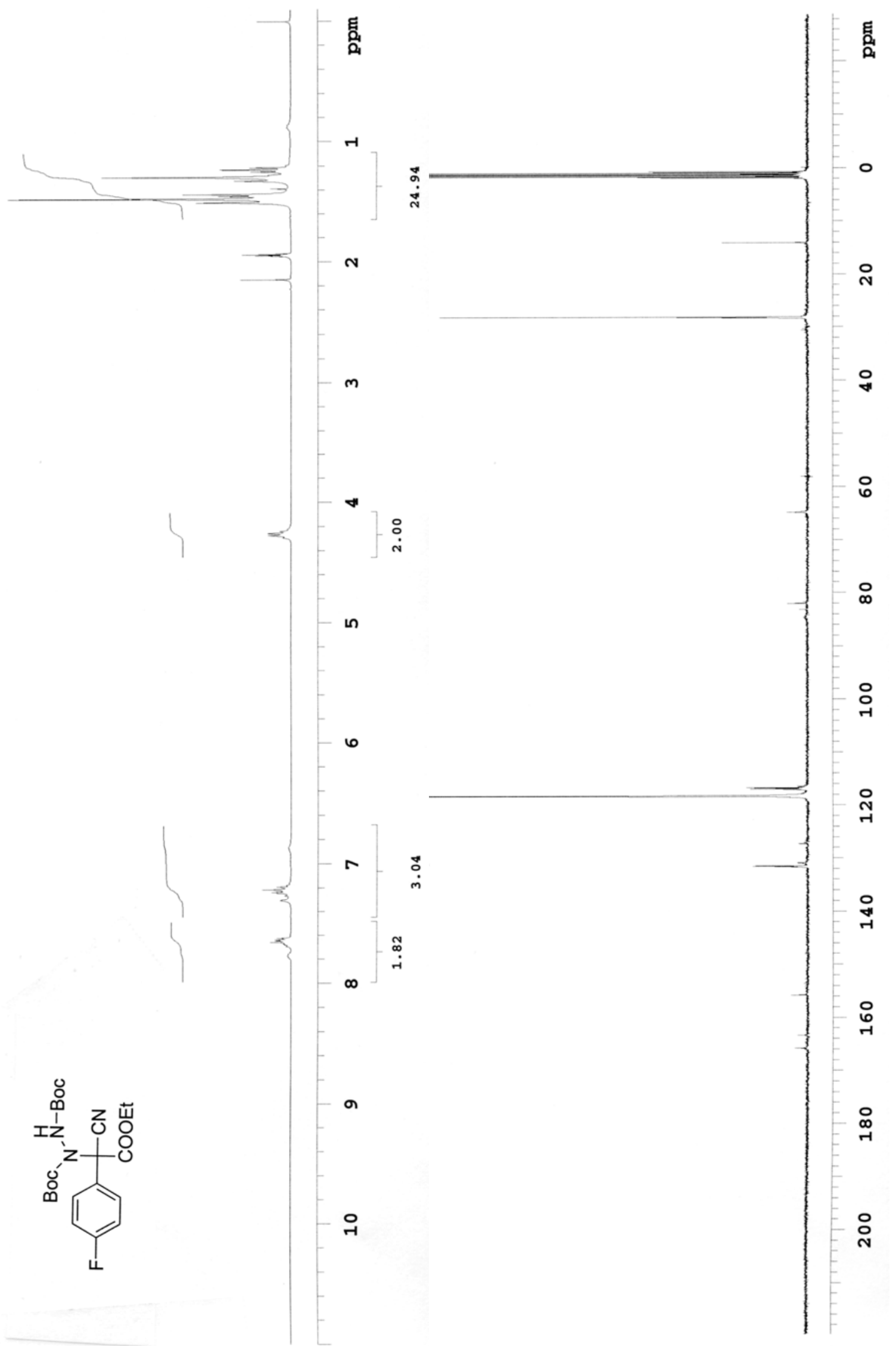


HPLC Conditions: Daicel Chirapak AD, hexanes:IPA 85:15, $1.0 \mathrm{ml} / \mathrm{min}$.<smiles>CCOC(=O)C(NC(=O)OCc1ccccc1)(NC(=O)OC(C)(C)C)C(C#N)(C(=O)OCc1ccccc1)c1ccc(Cl)cc1</smiles>

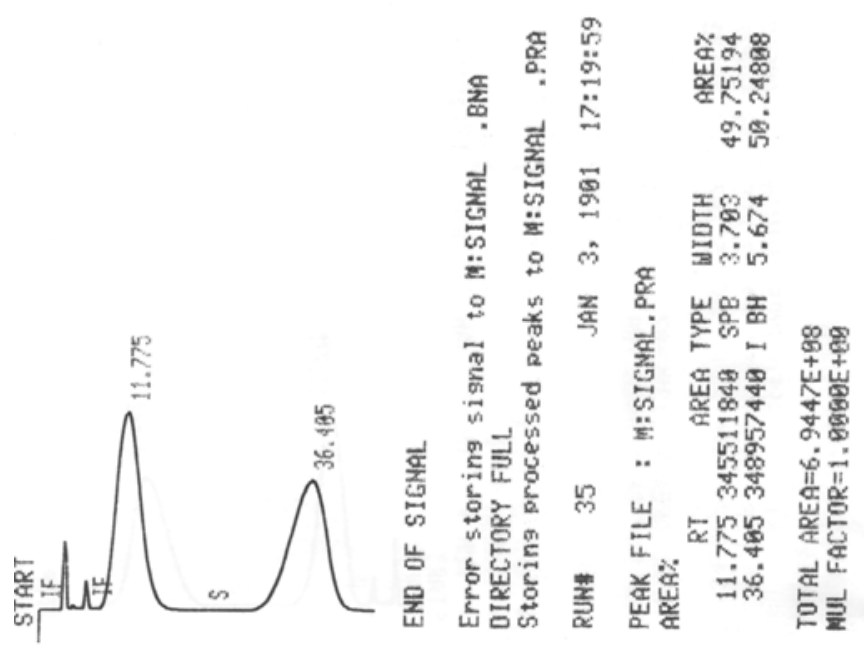

$(+)-5 c$

Product of Q-2c catalyzed reaction

\section{$(-)-5 c$}

Product of QD-2c catalyzed reaction
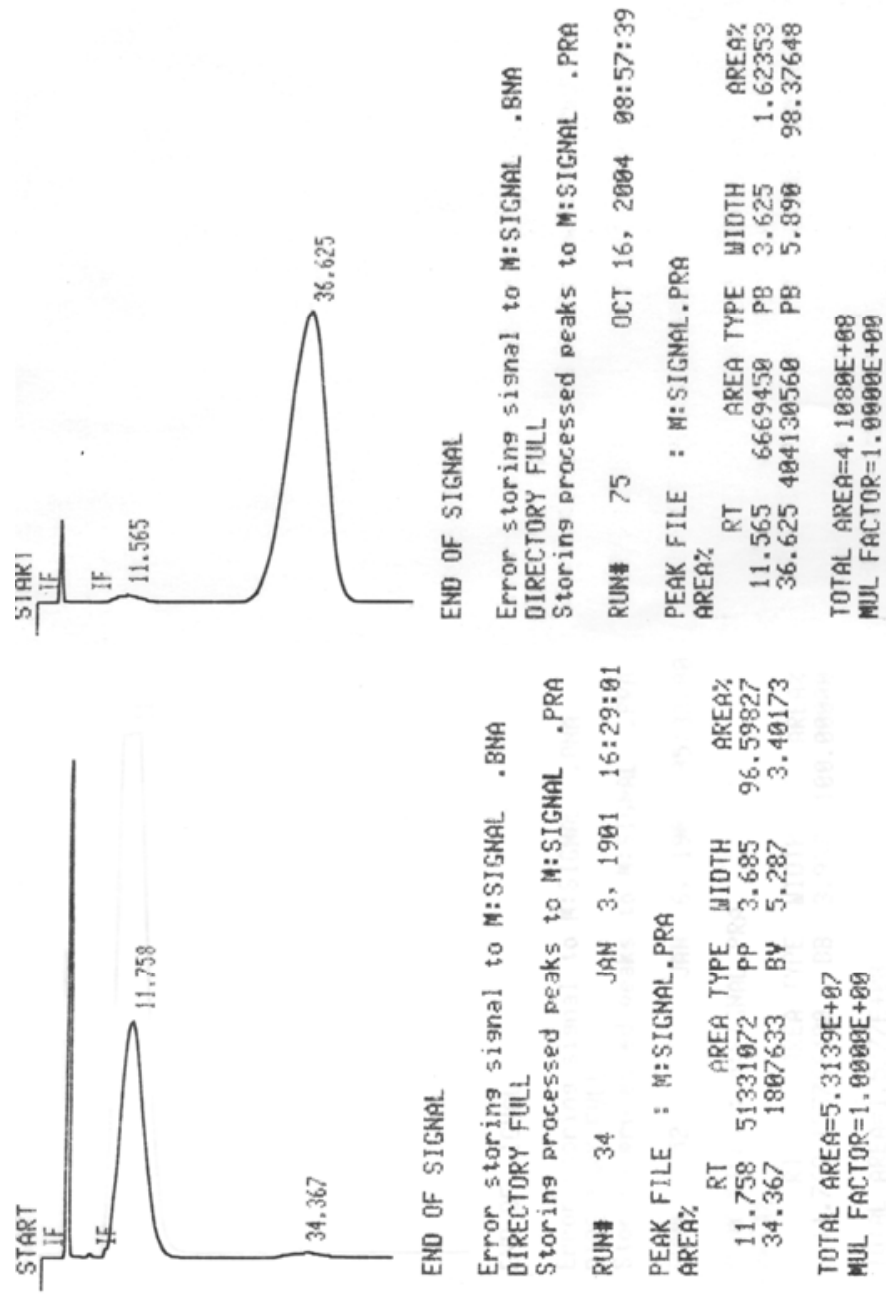


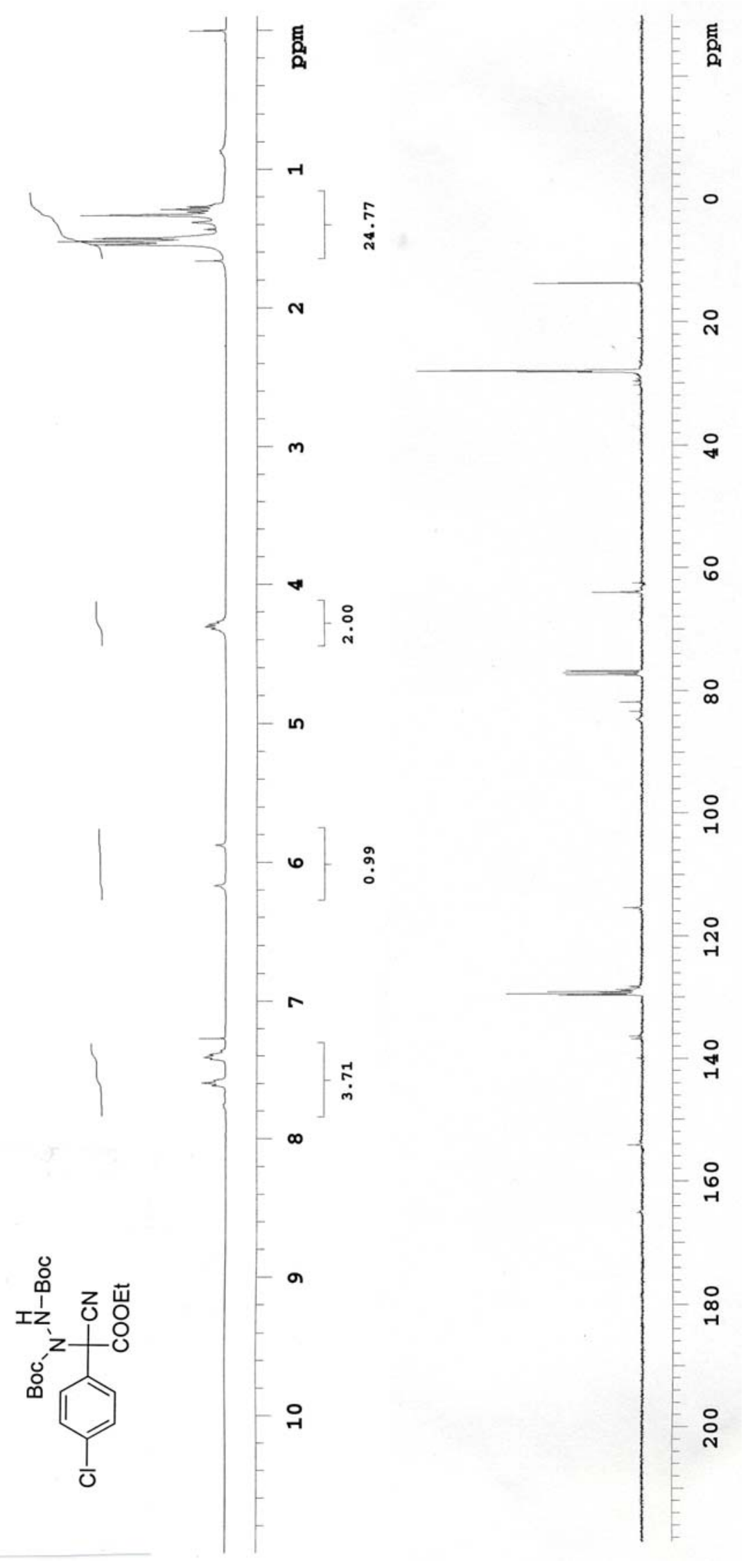


HPLC Conditions: Daicel Chirapak AD, hexanes:IPA 85:15, $1.0 \mathrm{ml} / \mathrm{min}$.

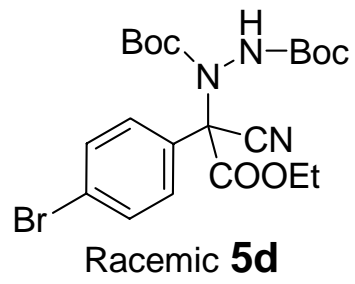

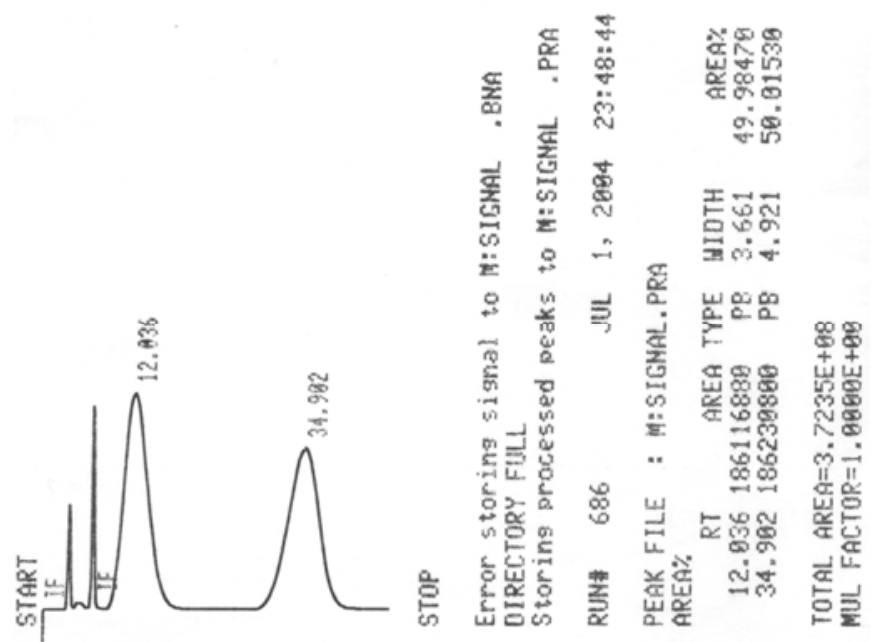

(+)-5d

Product of Q-2c catalyzed reaction

(-)-5d

Product of QD-2c catalyzed reaction
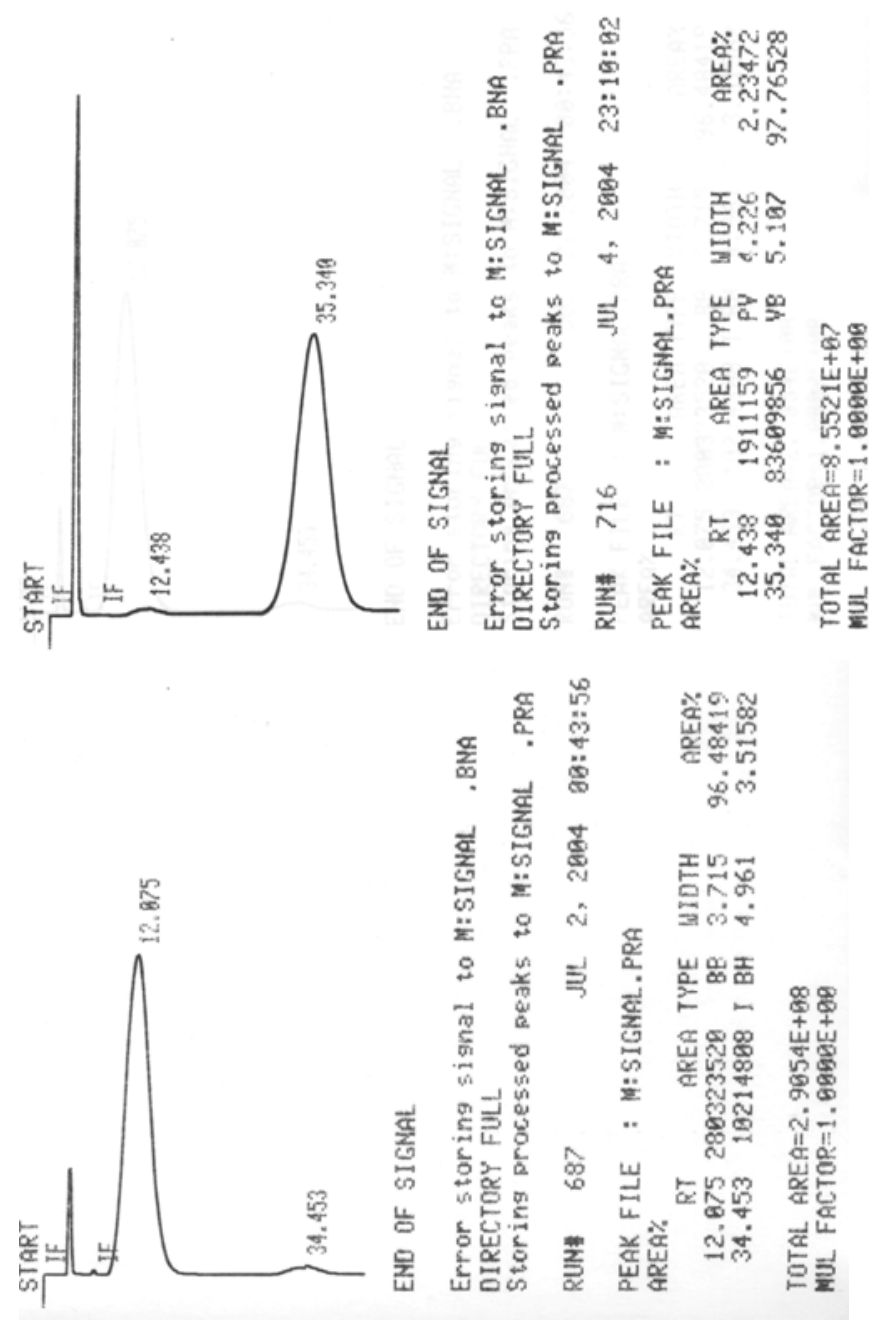


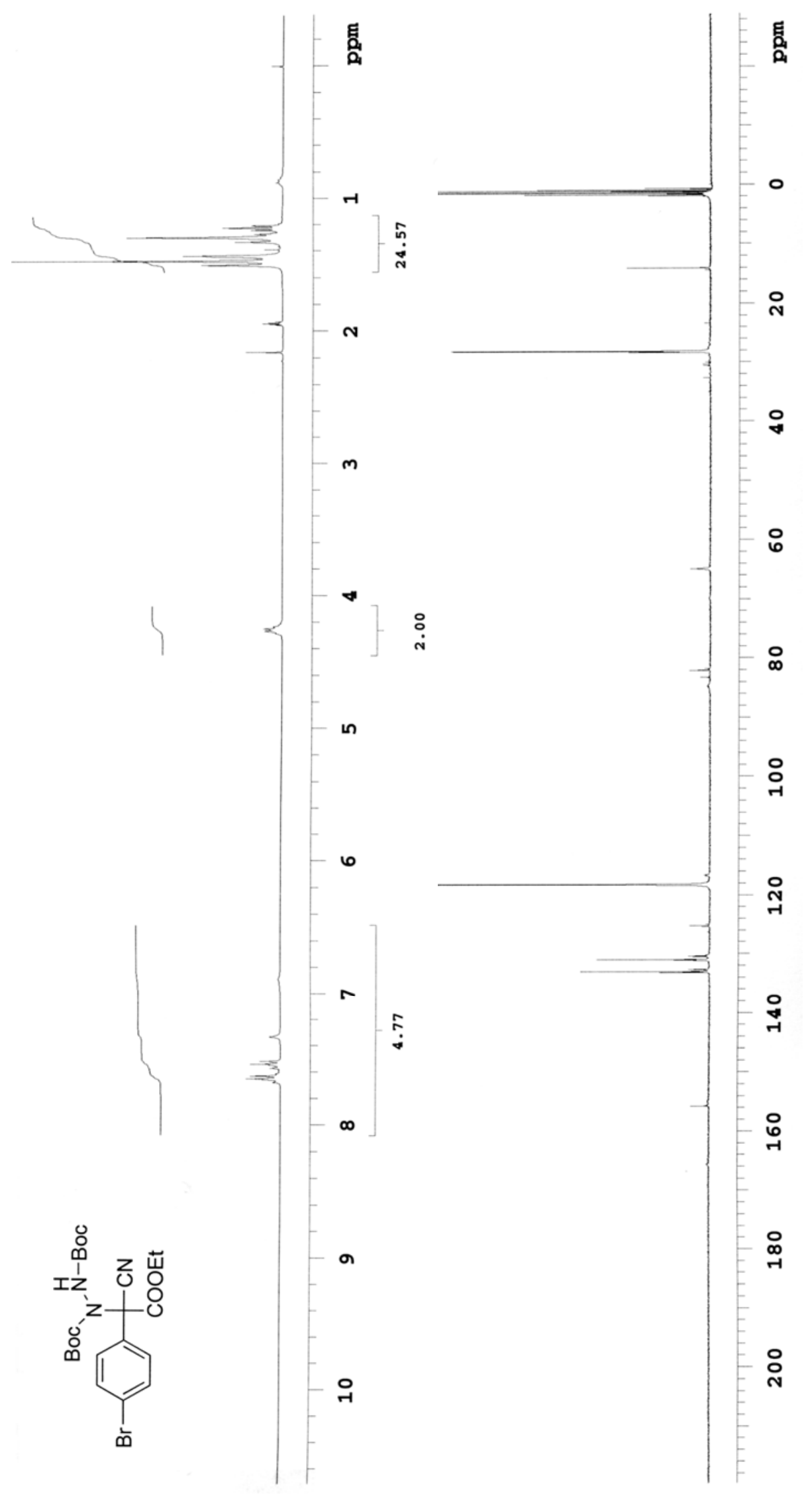


HPLC Conditions: Daicel Chirapak AD, hexanes:IPA 60:40, $1.0 \mathrm{ml} / \mathrm{min}$.<smiles>CCOC(=O)C(C)(C(=O)NNC(=O)OC(C)(C)C)C(NC(=O)OCc1ccccc1)(C(=O)OCC)c1cccc2ccccc12</smiles>

Racemic 5e

$(+)-5 e$

Product of Q-2c catalyzed reaction

$(-)-5 e$

Product of QD-2c catalyzed reaction
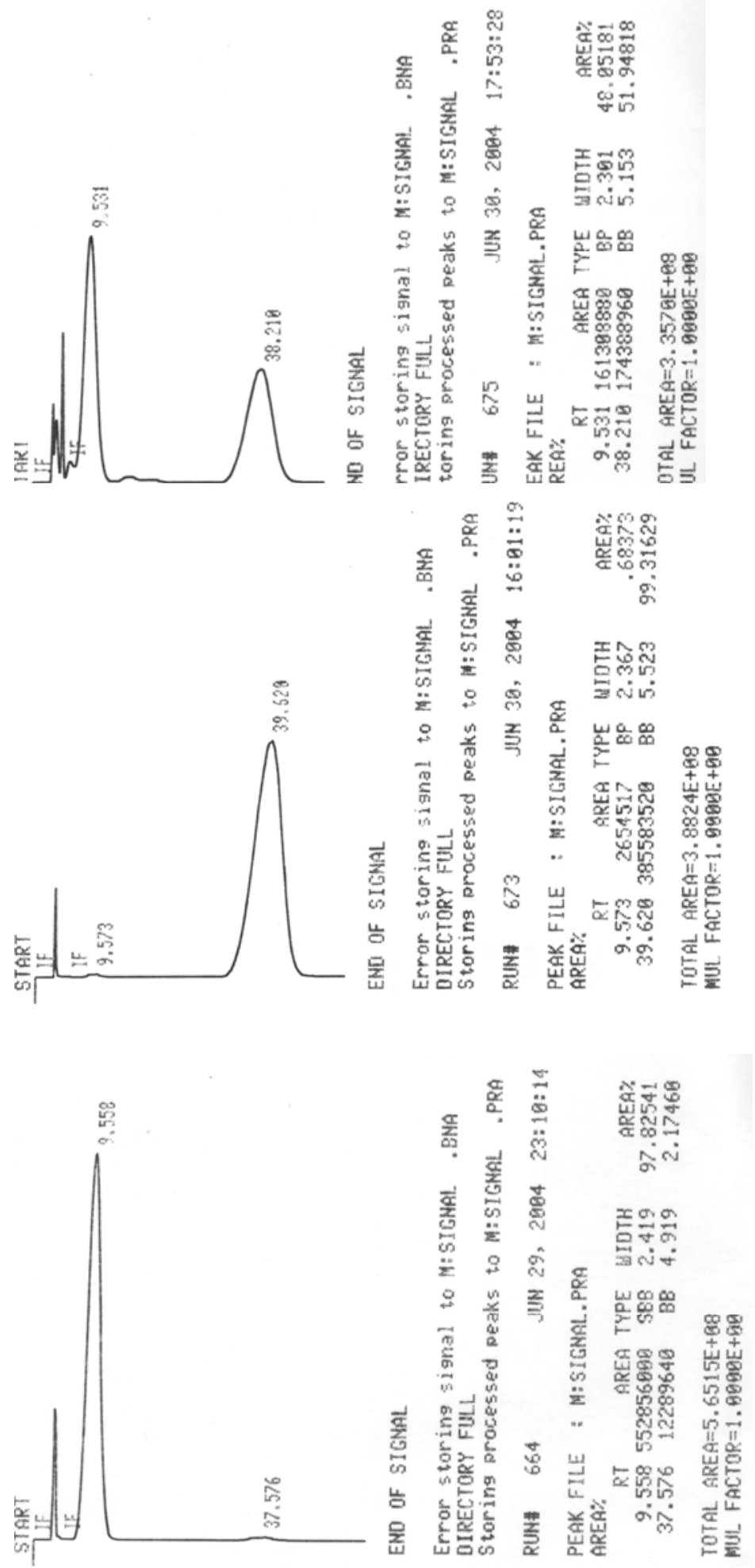


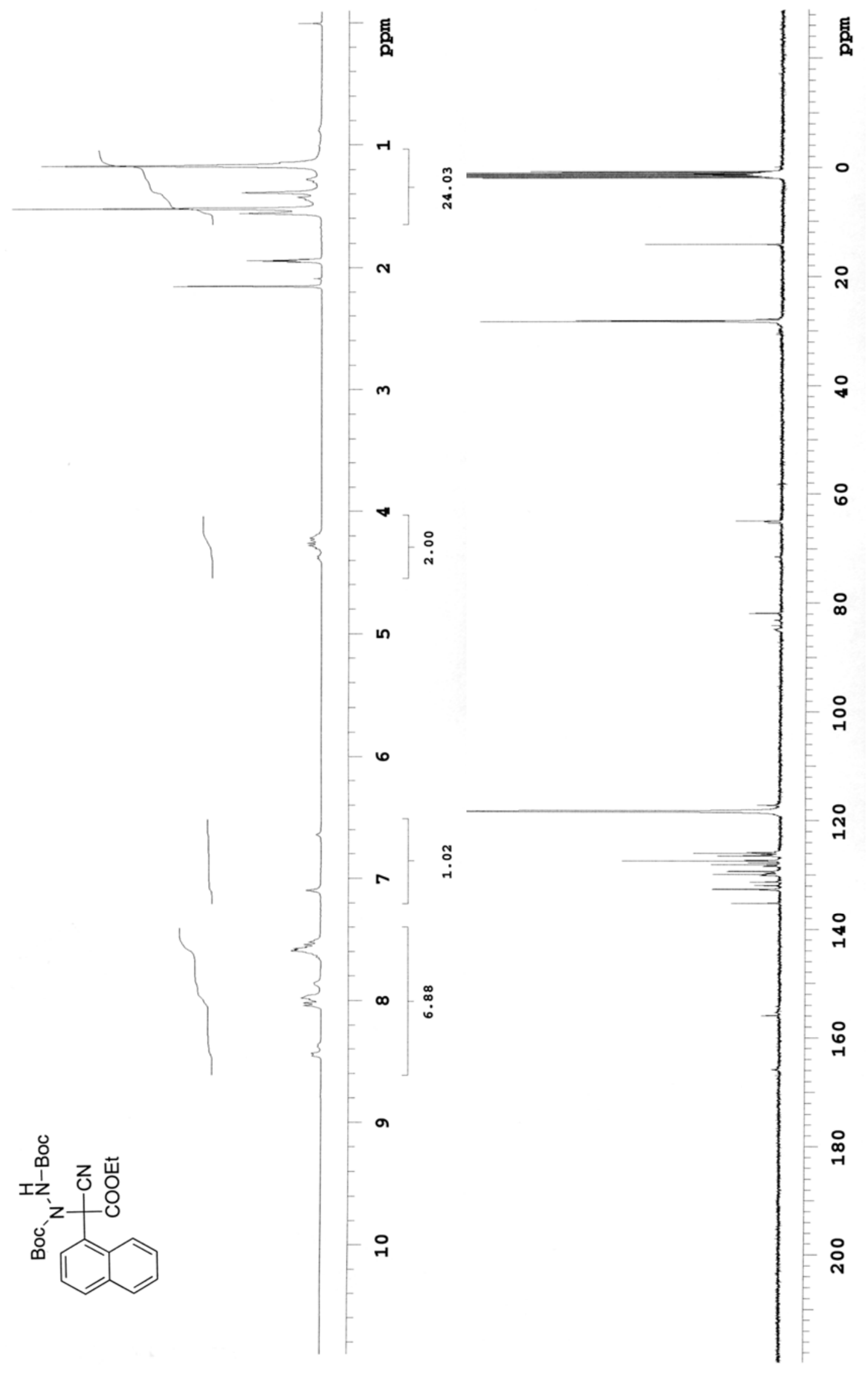


HPLC Conditions: Daicel Chirapak AD, hexanes:IPA 85:15, $1.0 \mathrm{ml} / \mathrm{min}$.
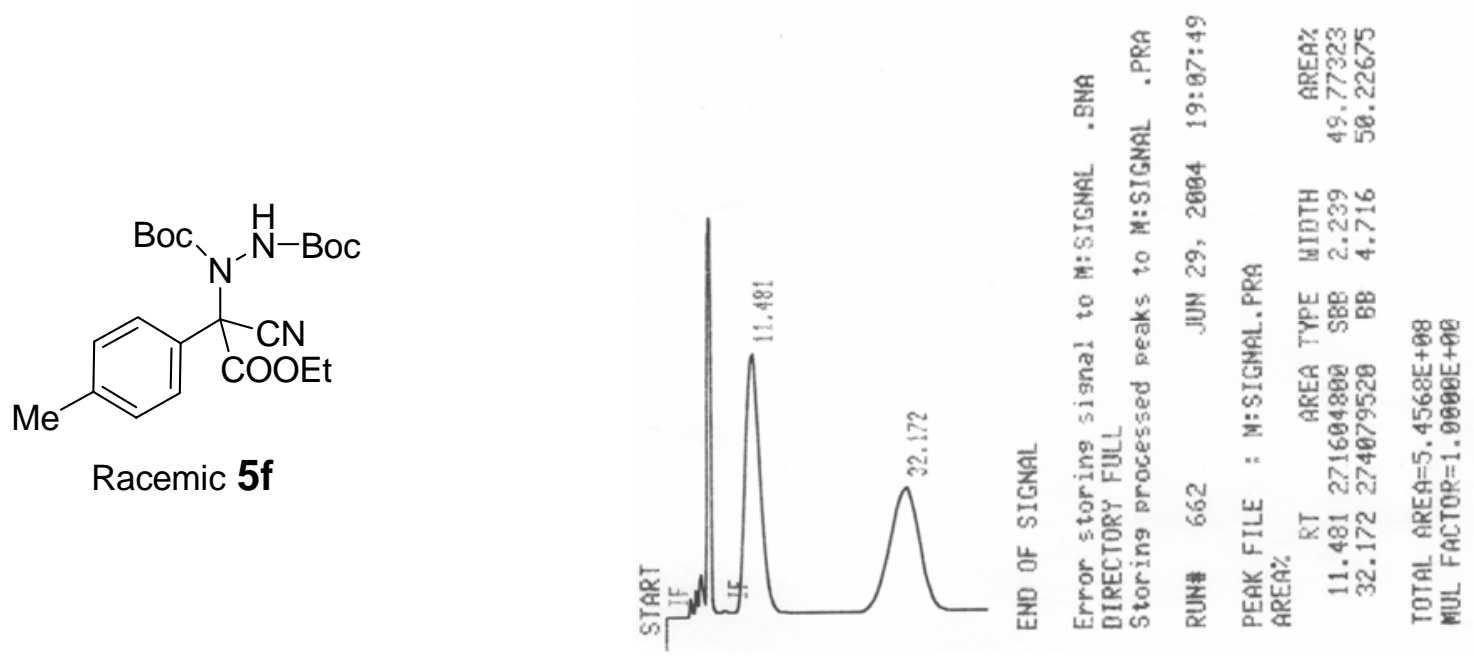

$(+)-5 f$

Product of Q-2c catalyzed reaction

$(-)-5 f$

Product of QD-2c catalyzed reaction
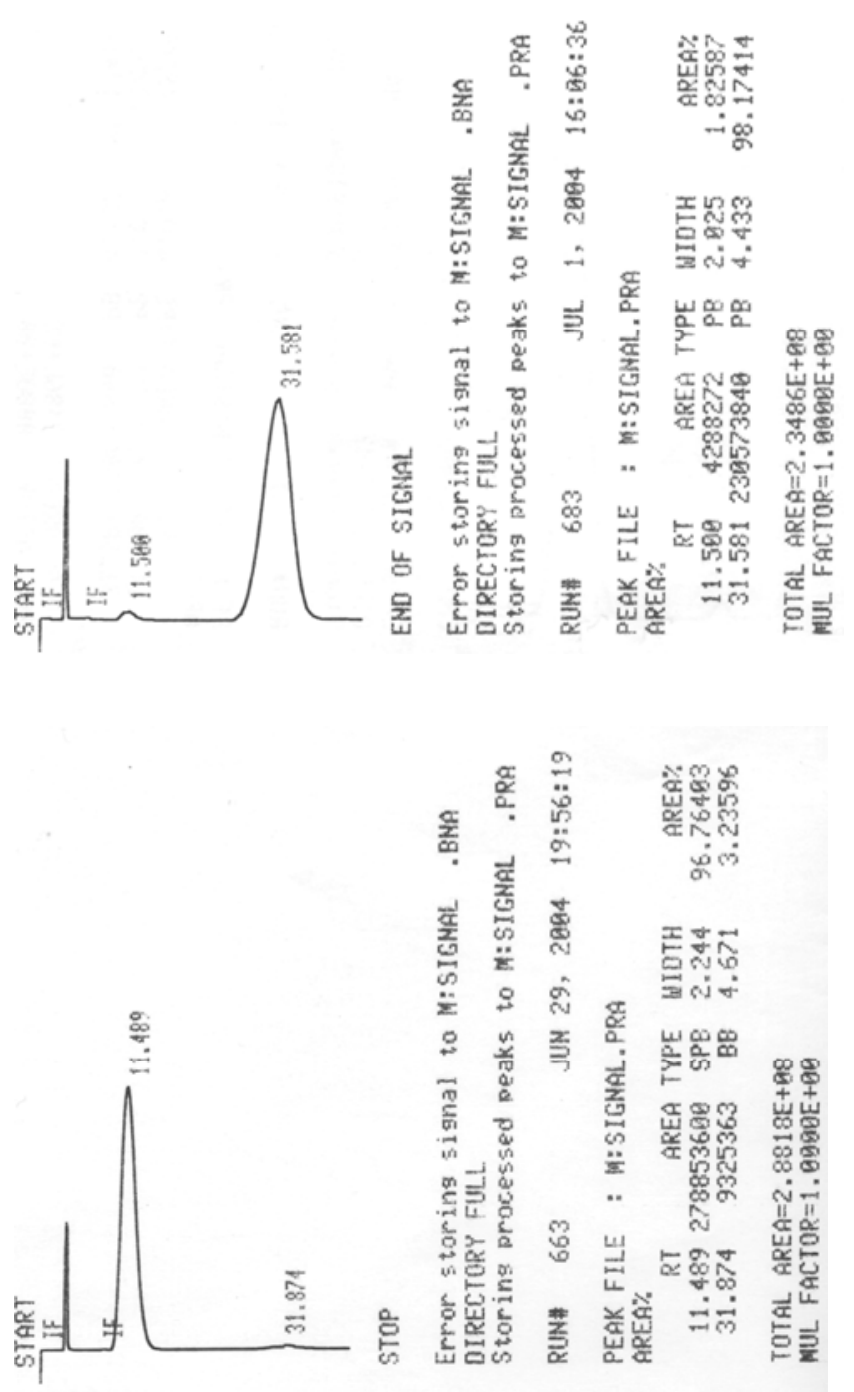


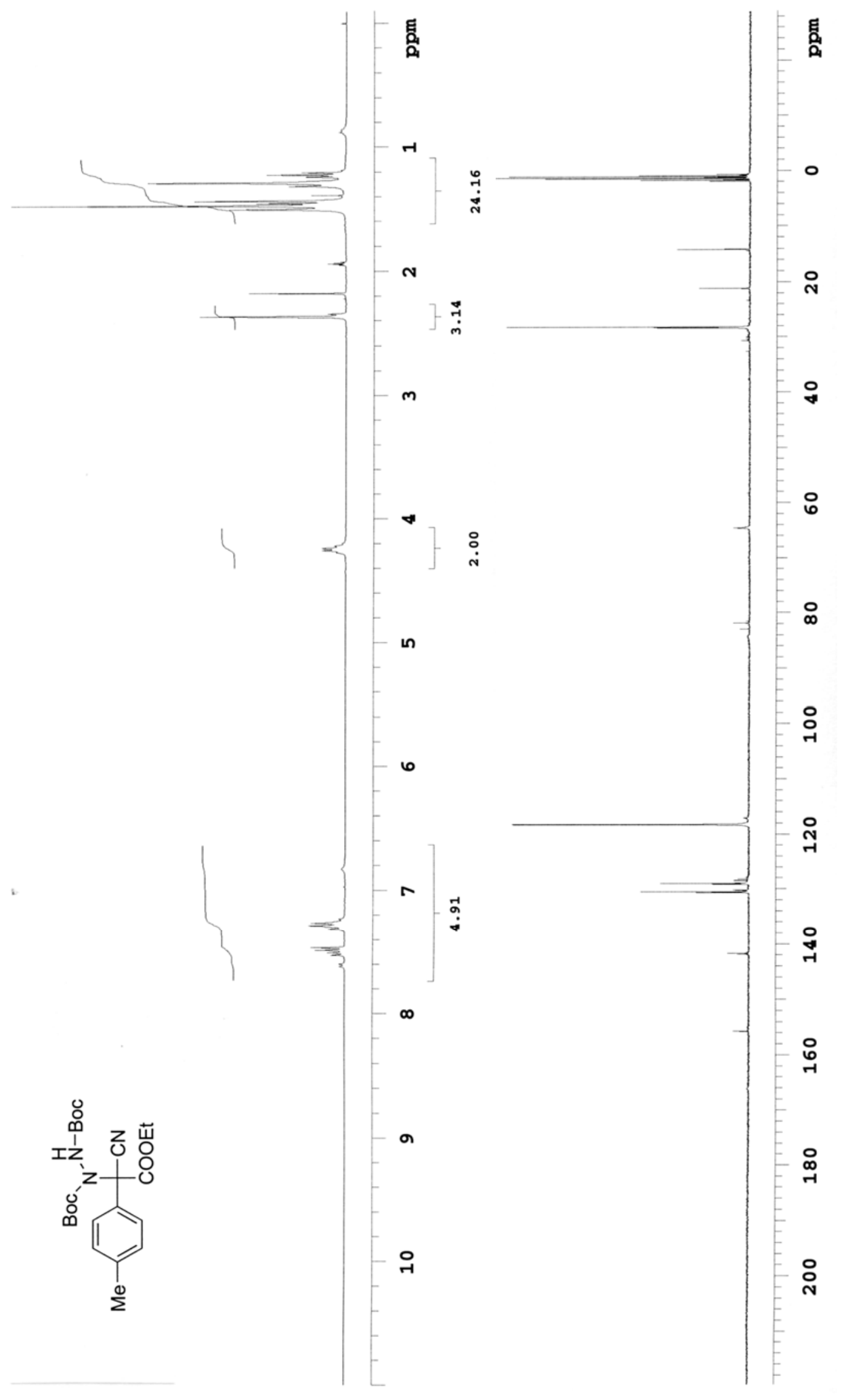


HPLC Conditions: Daicel Chirapak OD, hexanes:IPA 97:3, 1.0 ml/min.<smiles>CCOC(=O)C(C)(C(=O)OCc1ccccc1)N(NC(=O)OCc1ccccc1)C(=O)OC(C)(C)C</smiles>

$(+)-5 g$

Product of Q-2c

catalyzed reaction
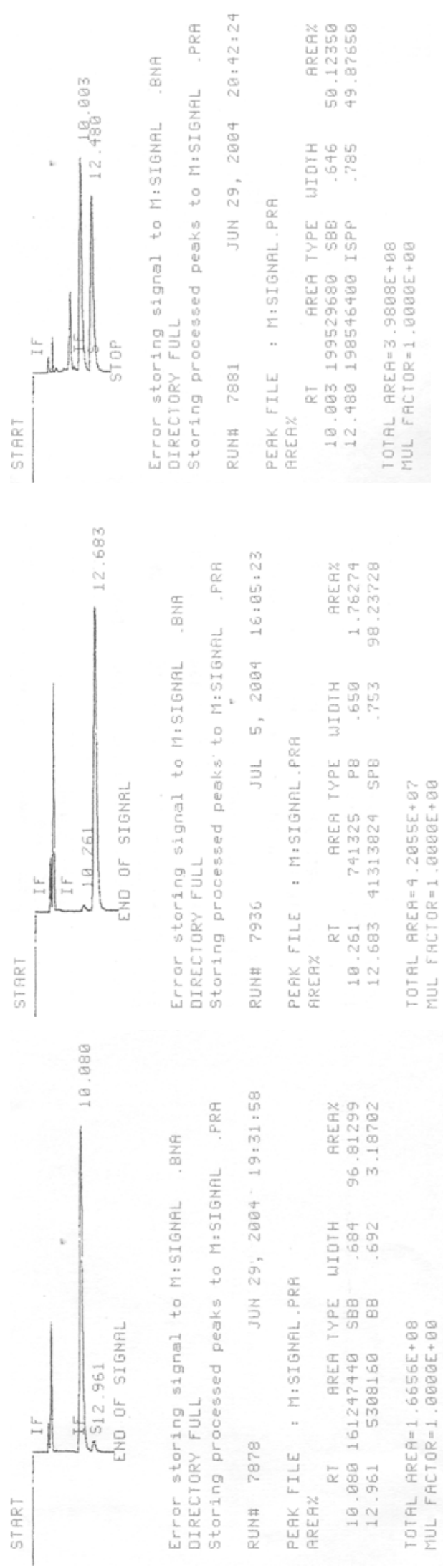


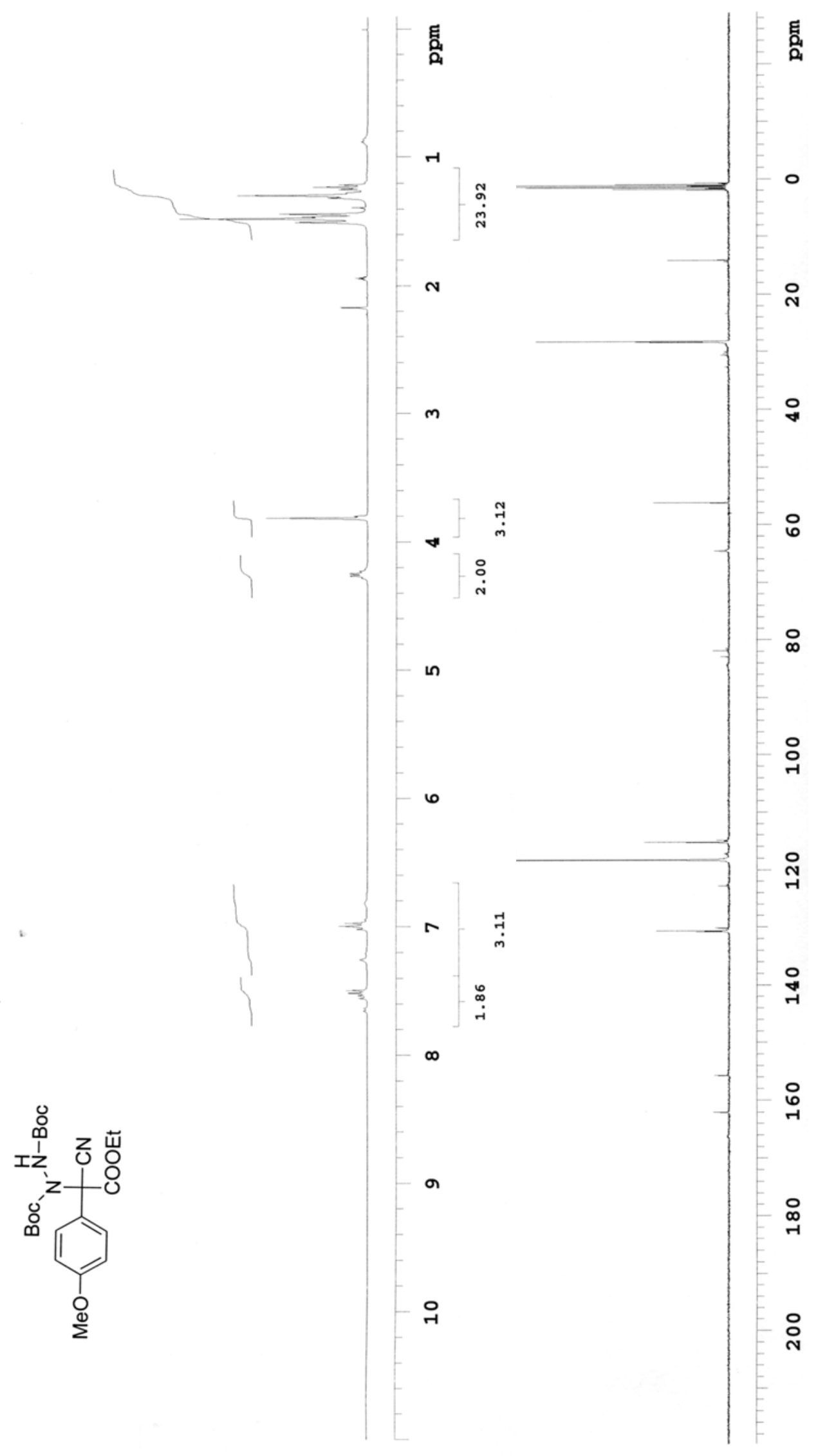


HPLC Conditions: Daicel Chirapak AS, hexanes:IPA 75:25, $1.0 \mathrm{ml} / \mathrm{min}$.<smiles>[R20]C(=O)NN(C(=O)OCC)C(C)(C(=O)OCC)C(C)(C(=O)OCc1ccccc1)c1ccc(Br)cc1</smiles>

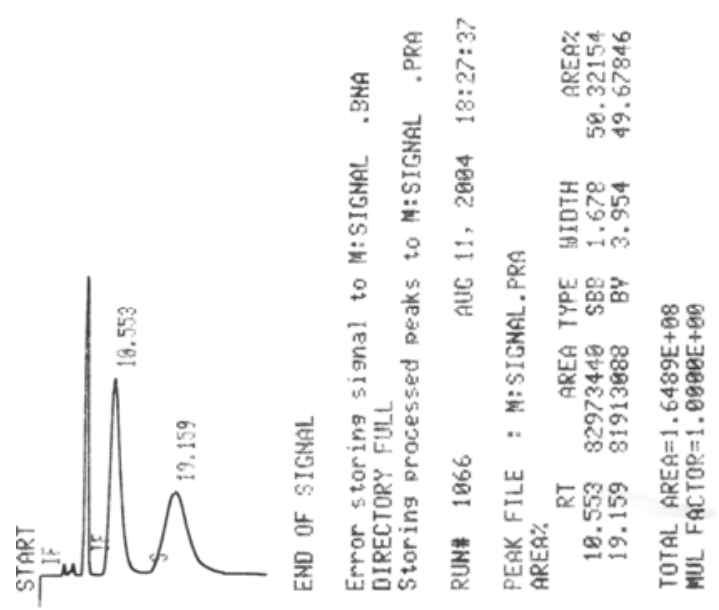

$(+)-5 h$

Product of Q-2c catalyzed reaction

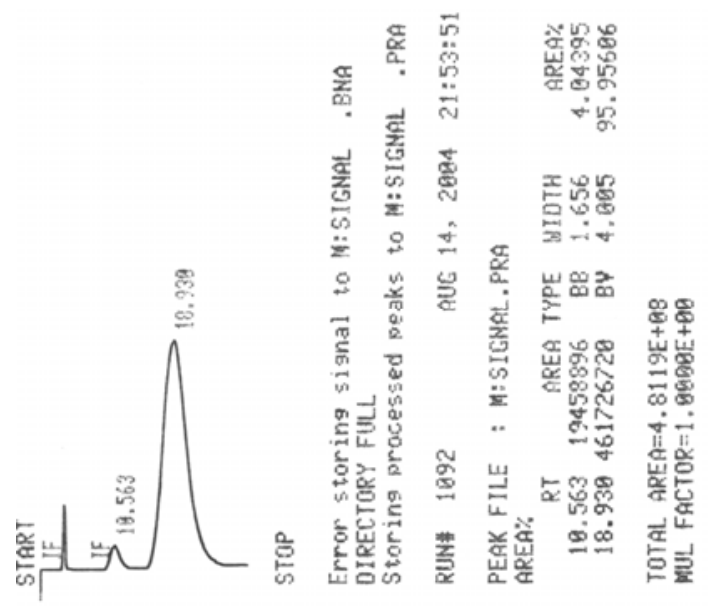

$(-)-5 h$

Product of QD-2c catalyzed reaction

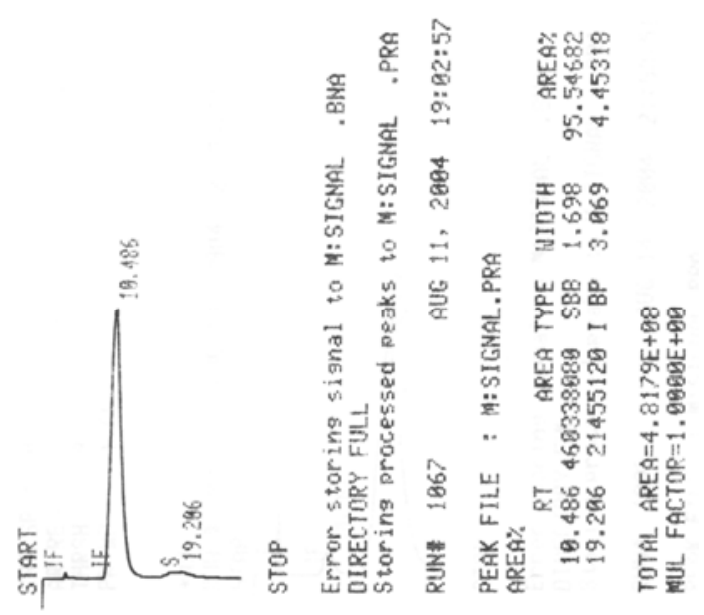



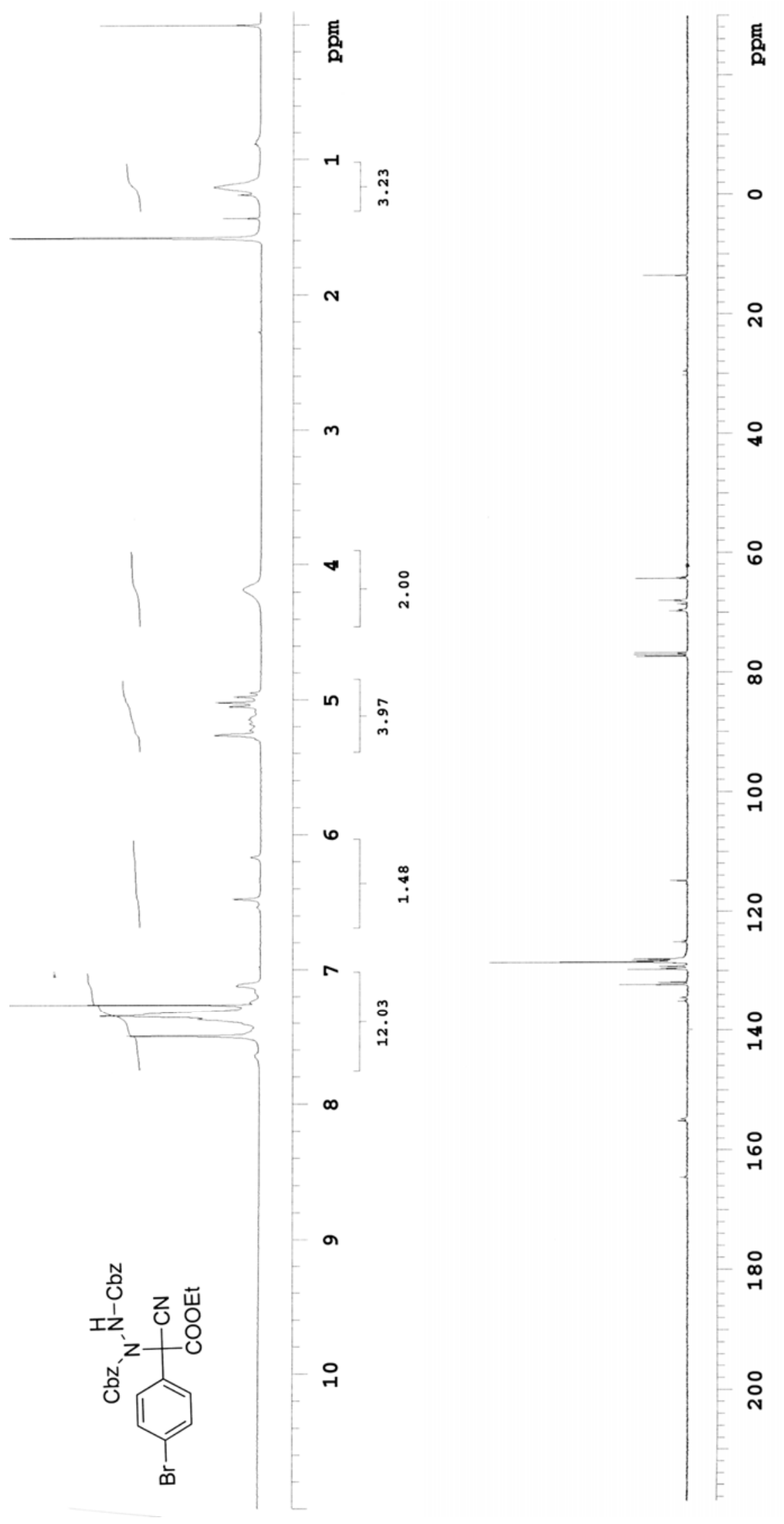
HPLC Conditions: Daicel Chirapak OD, hexanes:IPA 90:10, $1.0 \mathrm{ml} / \mathrm{min}$.<smiles>CCOC(=O)C(C)(C#N)C(NC(=O)OCc1ccccc1)(NC(=O)OCc1ccccc1)C(=O)OCc1ccccc1</smiles>

Racemic 5i

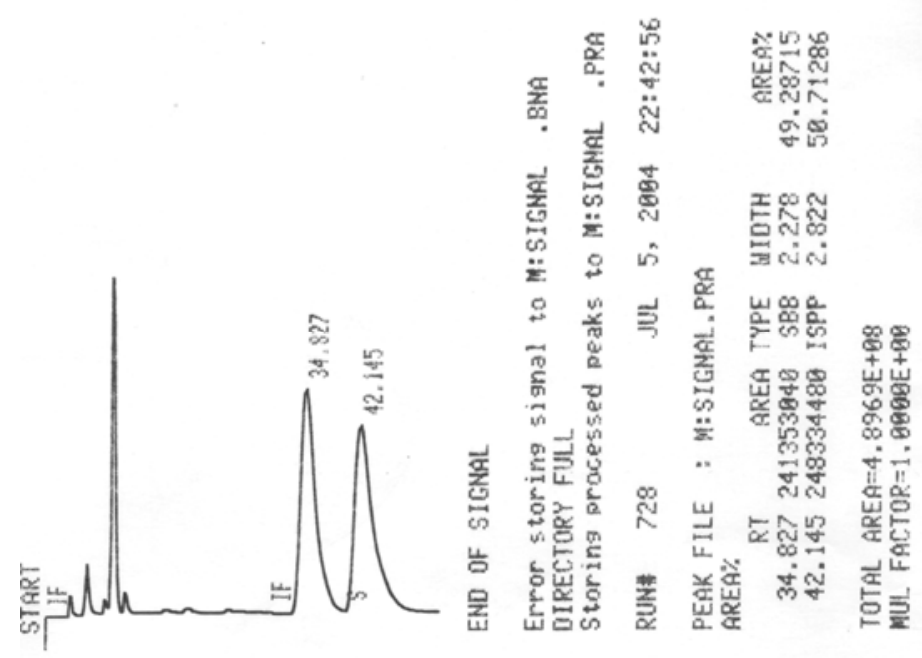

$(+)-5 i$

Product of Q-2c catalyzed reaction

\section{$(-)-5 i$}

Product of QD-2c catalyzed reaction
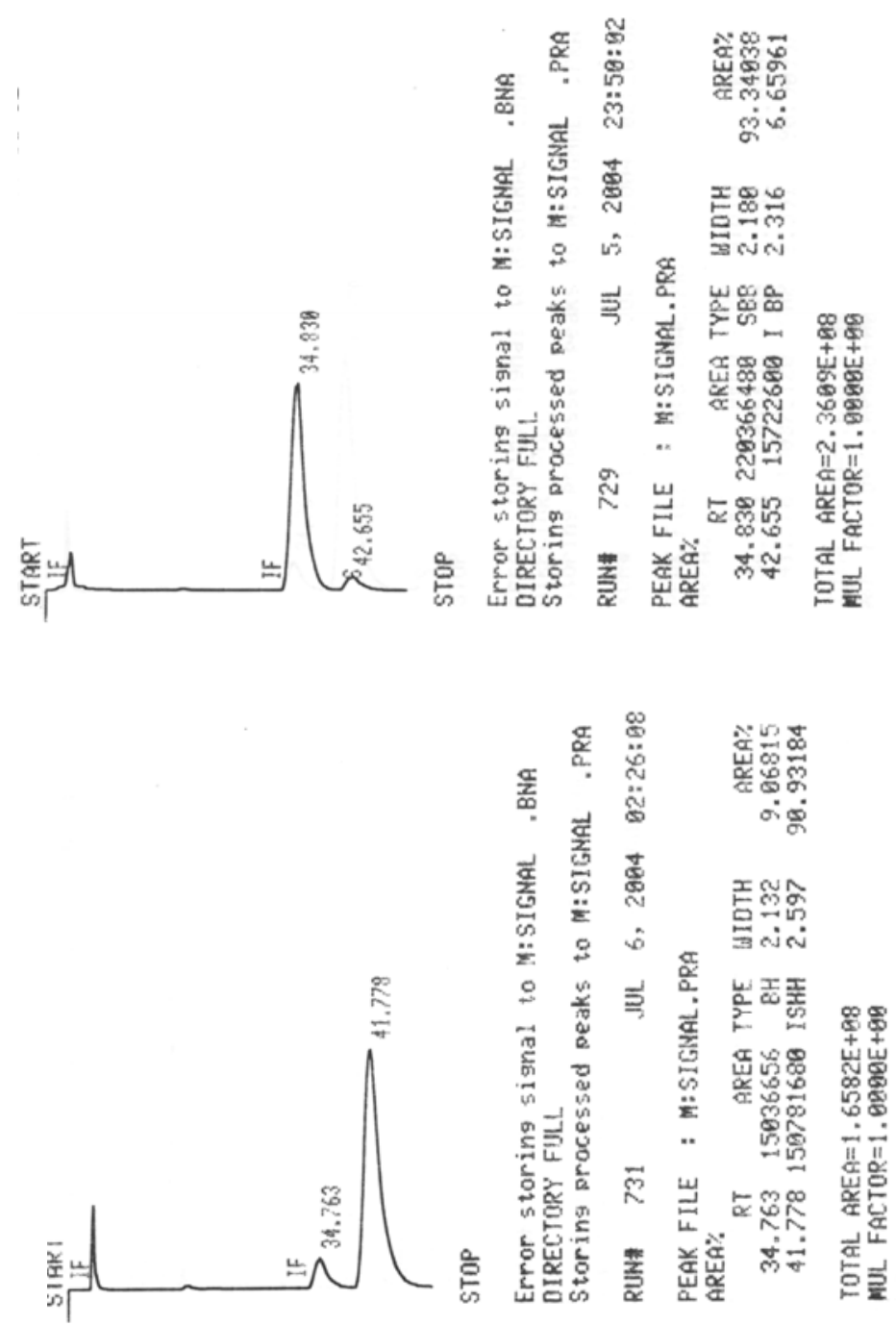


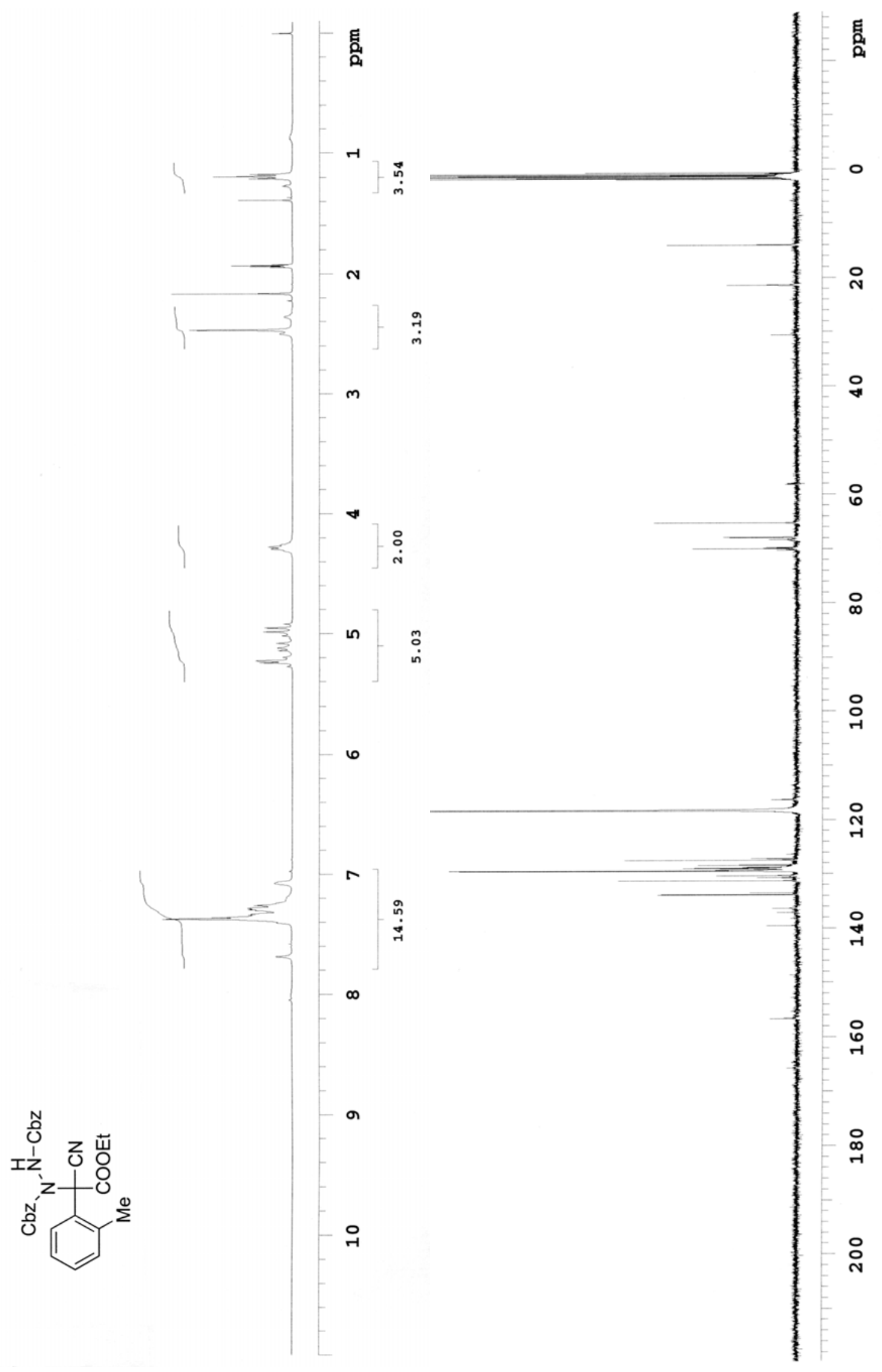


HPLC Conditions: Daicel Chirapak OD, hexanes:IPA 93:7, $1.0 \mathrm{ml} / \mathrm{min}$.<smiles>CCOC(=O)NC(C)(C(=O)OCC)N(C)C1CCCCC1</smiles>

Racemic 5j

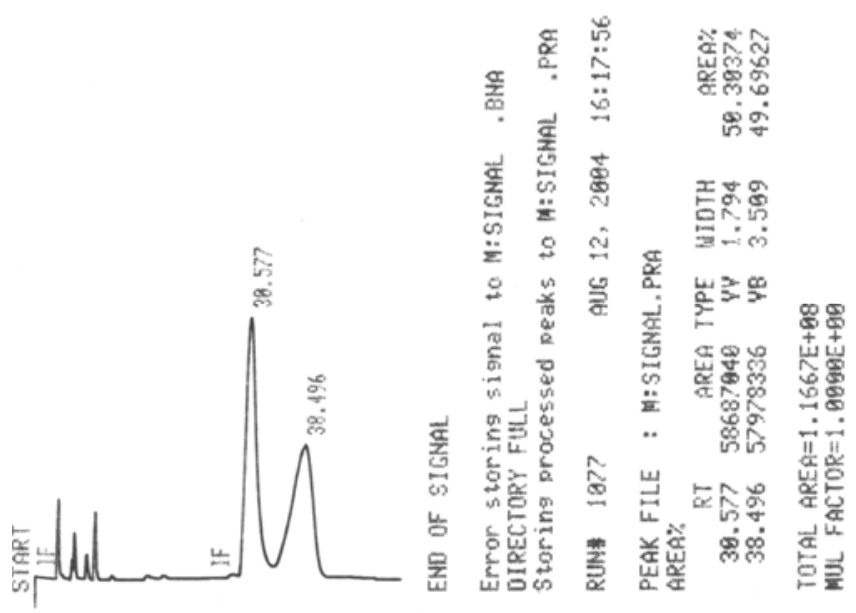

$(-)-5 j$

Product of Q-2c catalyzed reaction
$(+)-5 j$

Product of QD-2c catalyzed reaction
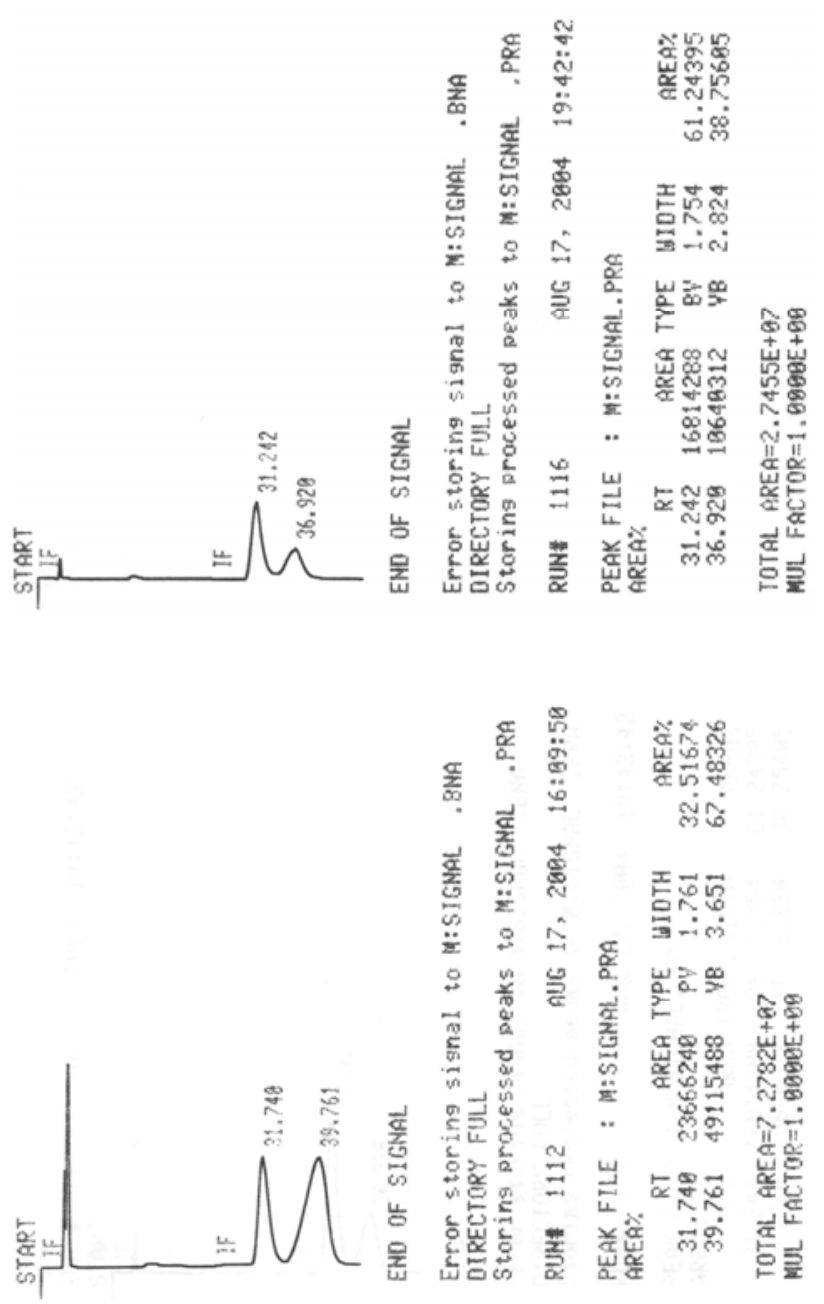

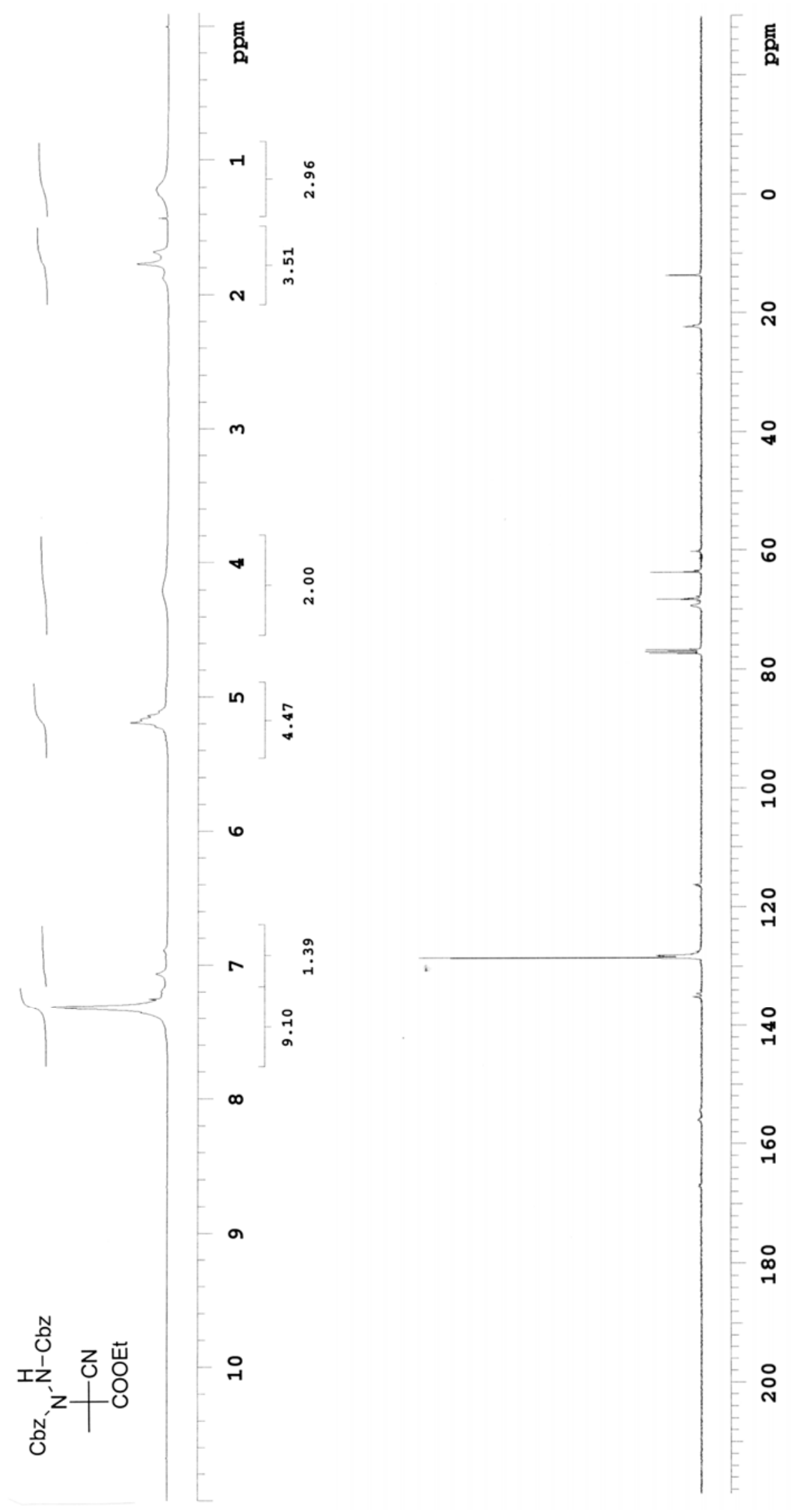
X-Ray Structure Determination. Single crystal of (-)-5c (prepared using catalyst QD-2c as described in this supporting information) suitable for X-ray diffraction measurement was obtained by recrystallization from hexanes/diethyl ether.

Data were collected using a Bruker SMART CCD (charge coupled device) based diffractometer equipped with an Oxford Cryostream low-temperature apparatus operating at $193 \mathrm{~K}$. A suitable crystal was chosen and mounted on a glass fiber using grease. Data were measured using omega scans of $0.3^{\circ}$ per frame for 45 seconds, such that a hemisphere was collected. A total of 1271 frames were collected with a maximum resolution of $0.76 \AA$. The first 50 frames were recollected at the end of data collection to monitor for decay. Cell parameters were retrieved using $\mathrm{SMART}^{3}$ software and refined using SAINT on all observed reflections. Data reduction was performed using the SAINT software ${ }^{4}$ which corrects for Lp and decay. The structures are solved by the direct method using the SHELXS- $97^{5}$ program and refined by least squares method on $\mathrm{F}^{2}$, SHELXL-97, ${ }^{6}$ incorporated in SHELXTL-PC V 6.10. ${ }^{7}$

The structure was solved in the space group $\mathrm{P} 22_{1} 2_{1} 2_{1}$ (\# 19) by analysis of systematic absences. All non-hydrogen atoms are refined anisotropically. Hydrogens were found by difference fouier methods and refined isotropicaly. The crystal used for the diffraction study showed no decomposition during data collection. All drawing are done at 50\% ellipsoids.

Acknowledgement. The CCD based x-ray diffractometer at Harvard University was purchased through NIH grant (1S10RR11937-01).

\footnotetext{
${ }^{1}$ Kaiser, E. M.; Solter, L. E.; Schwarz, R. A.; Beard, R. D.; Hauser, C. R. J. Am. Chem. Soc. 1971, 93, 4237.

${ }^{2}$ Albarella, J. M. J. Org. Chem. 1977, 42, 2009.

${ }^{3}$ SMART V 5.625 (NT) Software for the CCD Detector System; Bruker Analytical X-ray Systems, Madison, WI (2001).

${ }^{4}$.SAINT V 6.22 (NT) Software for the CCD Detector System Bruker Analytical X-ray Systems, Madison, WI (2001).

${ }^{5}$ Sheldrick, G. M. SHELXS-90, Program for the Solution of Crystal Structure, University of Göttingen, Germany, 1990.

${ }^{6}$ Sheldrick, G. M. SHELXL-97, Program for the Refinement of Crystal Structure, University of Göttingen, Germany, 1997.

${ }^{7}$ SHELXTL 6.1 (PC-Version), Program library for Structure Solution and Molecular Graphics; Bruker Analytical X-ray Systems, Madison, WI (2000).

${ }^{\mathrm{a}}$ Obtained with graphite monochromated Mo K $(=0.71073 \AA)$ radiation. ${ }^{\mathrm{b}} R 1=\sum|| F_{\mathrm{o}} \mid-$ $\left|F_{\mathrm{c}}\right|\left|/ \sum\right| F_{\mathrm{o}} \mid .{ }^{\mathrm{c}} w R_{2}=\left\{\sum\left[w\left(F_{\mathrm{o}}{ }^{2}-F_{\mathrm{c}}\right)^{2} /\left\{\sum\left[w\left(F_{\mathrm{o}}\right)^{2}\right]\right\}^{1 / 2}\right.\right.$.
} 


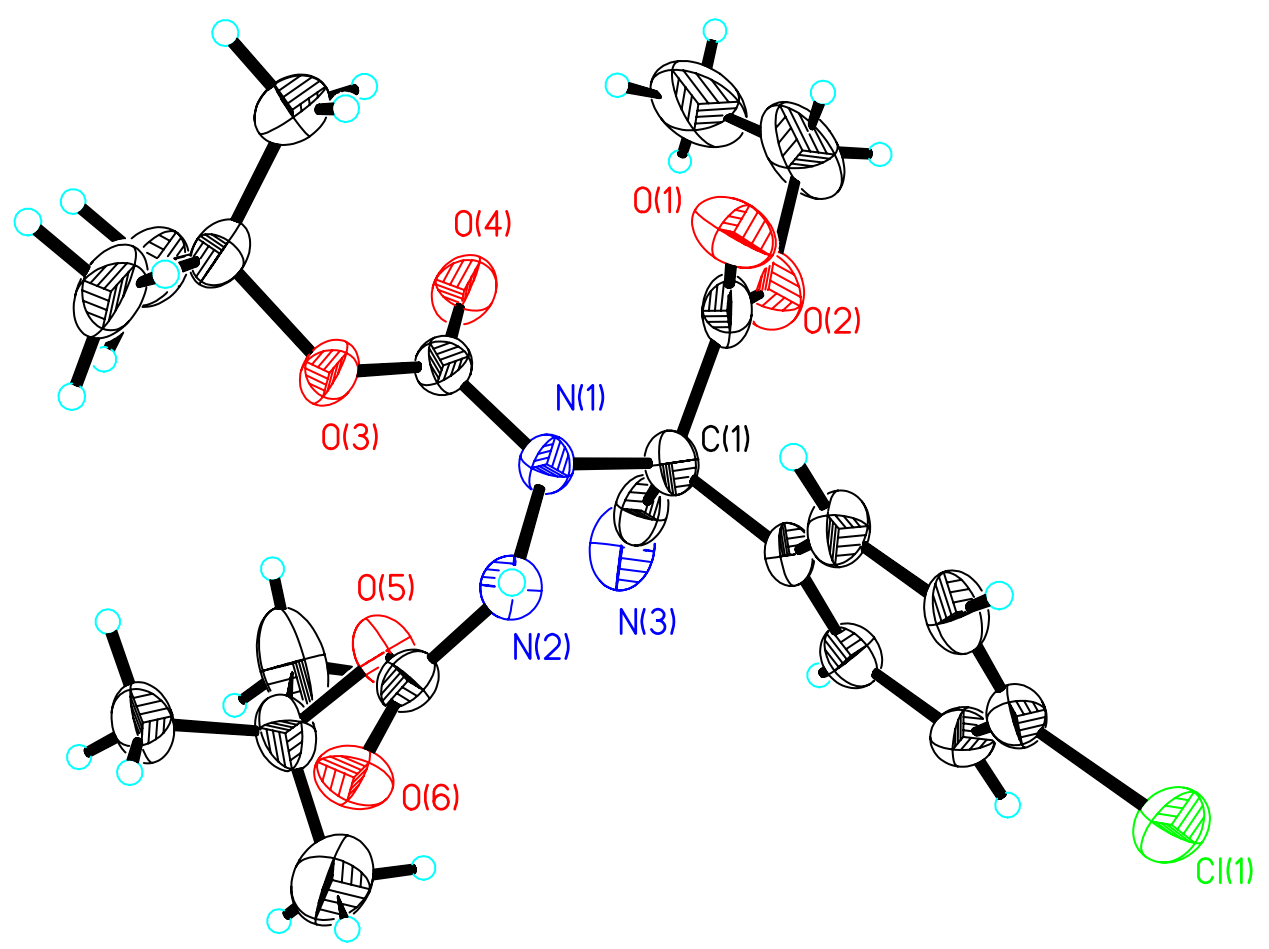

Figure 1. Molecular Structure of $\mathbf{5 c}$ (prepared using catalyst QD-2c as described in this supporting information) 